\title{
A Quantitative Model of Early Atherosclerotic Plaques Parametrized Using In Vitro Experiments
}

\author{
Moritz P. Thon - Hugh Z. Ford . \\ Michael W. Gee • Mary R. Myerscough
}

Received: date / Accepted: date

\begin{abstract}
There is a growing number of studies that model immunological processes in the artery wall that lead to the development of atherosclerotic plaques. However, few of these models use parameters that are obtained from experimental data even though data-driven models are vital if mathematical models are to become clinically relevant.

We present the development and analysis of a quantitative mathematical model for the coupled inflammatory, lipid and macrophage dynamics in early atherosclerotic plaques. Our modeling approach is similar to the biologists' experimental approach where the bigger picture of atherosclerosis is put together from many smaller observations and findings from in vitro experiments. We first develop a series of three simpler submodels which are least-squares fitted to various in vitro experimental results from literature. Subsequently, we use these three submodels to construct a quantitative model of the development of early atherosclerotic plaques.

We perform a local sensitivity analysis of the model with respect to its parameters that identifies critical parameters and processes. Further, we present a systematic analysis of the long-term outcome of the model which produces a characterization of the stability of model plaques based on the rates of recruitment of low-density lipoproteins, high density lipoproteins and macrophages. The analysis of the model suggests that further experimental work quantifying the different fates of macrophages as a function of cholesterol load and the balance between free cholesterol and cholesterol ester inside macrophages may
\end{abstract}

Moritz P. Thon · Michael W. Gee (凶)

Mechanics \& High Performance Computing Group, Technical University of Munich, Parkring 35, 85748 Garching b. München, Germany

E-mail: gee@tum.de

Hugh Z. Ford · Mary R. Myerscough

School of Mathematics and Statistics F07, University of Sydney, New South Wales 2006, Australia 
give valuable insight into long-term atherosclerotic plaque outcomes. This model is an important step towards models applicable in a clinical setting.

Keywords atherosclerosis - plaque developement - quantitative model . parameter estimation $\cdot$ metabolic control analysis $\cdot$ stability analysis

\section{Introduction}

Atherosclerosis is a chronic inflammatory disease of the artery wall [58, 18] and is a significant contributor to worldwide morbidity and mortality. We present a quantitative mathematical model which addresses whether early stage plaques will grow or regress based on the rates of low-density lipoproteins (LDL) influx, high density lipoproteins (HDL) influx and macrophage recruitment into the artery wall. These quantities reflect the serum level of LDL and HDL in the bloodstream and the magnitude of wall shear stress exerted on the vascular wall by blood flow. HDL and LDL are routinely measured from patient blood samples and hemodynamic properties can be accurately estimated using threedimensional simulations of blood flow through patient-specific geometries of vasculature [12,19,44, 15.

There is an increasing demand for the quantification of inflammatory processes, particularly in atherosclerosis [51,73,21]. Even though a variety of mathematical models for many aspects of atherosclerosis exist, see 51. and therein, one of the major problems is that the models' parameters are commonly unknown and can often only be estimated very loosely. Yet, there has been remarkably little work done resolving this issue and quantifying crucial immunological processes in atherosclerosis. To create this model we have used published experimental results to obtain a comprehensive set of parameters to model immunological and biochemical processes that occur within the artery wall in the early stages of plaque formation. Other researchers have found parameters for models that focus on the biomechanical or perfusion properties of the artery walls $[12,19,44,80,55,76$. Although there is a growing body of work that models cellular and lipid dynamics inside the artery wall[ [50, 9, 2 , 21, 11, 28,7, 17,8, to our knowledge no complete set of experimental-derived parameters exists for any of these models. In order for mathematical models of inter- and intra-cellular dynamics to be clinically useful, these models need to be comprehensively and correctly parametrised from experimental data. This study is the first significant step in that endeavour.

Atherosclerosis is characterized by sterile inflammatory lesions in the artery wall called atherosclerotic plaques. Plaques form at sites where blood flow exerts a low wall shear stress on the wall [52]. This low wall shear stress causes a disruption of the endothelium that lines the lumen of the artery which increases the amount of LDL and HDL seeping into the vessel wall. LDL that seeps in from the bloodstream is retained in the artery wall and subjected to oxidative modifications which render it pro-inflammatory [74,65, 75. If a differentiation between oxidatively modified LDL and unmodified LDL is required, we will refer to them as oxidatively modified LDL (modLDL) and 
native LDL, respectively. The analogue holds for HDL. Accruing modLDL in the artery wall triggers an inflammatory response that draws in circulating monocytes from the bloodstream which differentiate into macrophages [14, 34. Monocyte-derived macrophages rapidly populate inflamed tissue, actively ingest noxious extracellular substances such as modLDL and necrotic debris, undergo controlled cell death (apoptosis) and consume other dead and dying macrophages (efferocytosis) [46,24]. Macrophages are at the crux of disease pathogenesis and resolution; they promote inflammation resolution by removing pro-inflammatory materials but can also drive further inflammation in response to modLDL [25, 46]. The inflammatory response is sustained by the persistent recruitment and modification of LDL and complicated by cholesterol that is present in LDL [36, 67.

Cholesterol uptake by macrophages via LDL and cholesterol efflux to HDL is tightly regulated by each macrophage to maintain an intracellular cholesterol content between essential and cytotoxic limits [3. This balance is disrupted in plaque macrophages due to the active consumption of modLDL [31, poor access to HDL [53] and efferocytosis which recycles cholesterol in apoptotic cells and retains it in the macrophage population. Macrophages thus accumulate cholesterol in excess which enhances pro-inflammatory signalling [78], apoptosis [16,77] and uncontrolled cell death (necrosis) [37,20,68]. These processes all contribute to a maladaptive inflammatory response.

In late but not early stage plaques, macrophage proliferation and emigration from the plaque may be enhanced so as to minimise cellular cholesterol content [54, 33, 38, 57,66. During the course of a human lifetime, the interplay between lipid and immune cell dynamics [45] drives characteristic changes in plaque constituents. These changes are used by clinicians to quantify and describe the disease 61]. The presence of large numbers of macrophages, a large pool of lipid-rich necrotic debris, called the necrotic core, and a thin covering between the plaque and bloodstream, called the fibrous cap, are the hallmarks of a dangerous plaque [72. The necrotic core is derived from macrophage necrosis in response to excessive cholesterol accumulation and post-apoptotic necrosis when apoptotic cells are not readily cleared by macrophages via efferocytosis [66, 46]. Growth of the necrotic core and migration of macrophages and other cells into the artery wall induces a swelling of the inner artery wall which leads to a expansion and remodeling of the artery wall [23]. In late stages this may result in a drastic narrowing of the lumen and the formation of a severe stenosis 35. If the fibrous cap ruptures due to the continues mechanical loading by the blood, the bloodstream is exposed to the thrombotic plaque constituents in the necrotic core. This triggers an occlusion event that results in serious clinical symptoms such as myocardial infarction, sudden cardiac death and stroke.

HDL has received considerable attention in therapy design as it offers a pathway for cholesterol removal from the plaque [4] and exerts anti-oxidant effects that reduces LDL modification in vitro [41. However, there is controversy over the efficacy of increasing HDL concentration in the blood serum as a therapy for late stage plaques but infusions of HDL mimetics show some promise [10,6] 
We construct a simple mathematical model of the early stages of atherosclerosis component-wise where each component is strongly informed by existing sets of in vitro experiments. The biological arguments and the chosen mathematical approach are philosophically the same, in that they rely on the idea that results from in vitro studies can be used to inform our understanding of what goes on in vivo.

The complete model is a quantitative and deterministic system of ordinary differential equations that describe the coupled inflammatory, lipid and macrophage dynamics inside the artery wall. We identify native LDL, modified LDL, native HDL, intracellular free cholesterol, intracellular cholesterol ester and macrophages as the key species in early atherosclerosis, see Fig 1 . The complete model tracks the number of LDL particles and the extent of their modification, the total number of HDL particles and the total number of macrophages and the extent of their intracellular cholesterol burden. Key and measurable processes during early stage atherosclerotic plaques include artery wall permeability with respect to LDL and HDL [70,64], LDL modification [27, 32, 41, 60, monocyte recruitment [29,1], monocyte-derived macrophage phagocytosis [27,32], intracellular lipid metabolism [4,5], macrophage apoptosis [77. and clearance of apoptotic macrophages which we assume are not defective 59. We use the complete model to forecast the constituents and properties of the plaque based on the recruitment rates of LDL, HDL and macrophages into the artery wall. We can use these predicted properties to determine when an early plaque will become unstable and grow unboundedly and hence become potentially dangerous and symptomatic [61.

This work contributes to a deeper understanding of the fundamental biological mechanisms behind the progression of atherosclerosis. Existing experimental results from literature are used to quantify processes that are essential to atherosclerotic plaque growth. The estimated physiological set of parameters is a major contribution of this work as it provides well-founded values for the rates of key processes that generate early atherosclerotic plaques.

The structure of the paper is as follows. In the Models and Methods section we present three mathematical submodels that reflect various in vitro experiments and each submodel is fitted to the relevant experimental results. The three submodels are combined to create a complete model that describes formation of early atherosclerotic plaques as they may occur in vivo. We perform a sensitivity analysis of the complete model with respect to its parameters and classify its long-term stability. In the Results section all computational results are presented and these are discussed and critically reflected in the Discussion section.

\section{Models and Methods}

The complete mathematical model is a combination of three simpler ordinary differential equation submodels which reflect various in vitro experiments. In all models, we use a continuum approach where the concentration of each species 


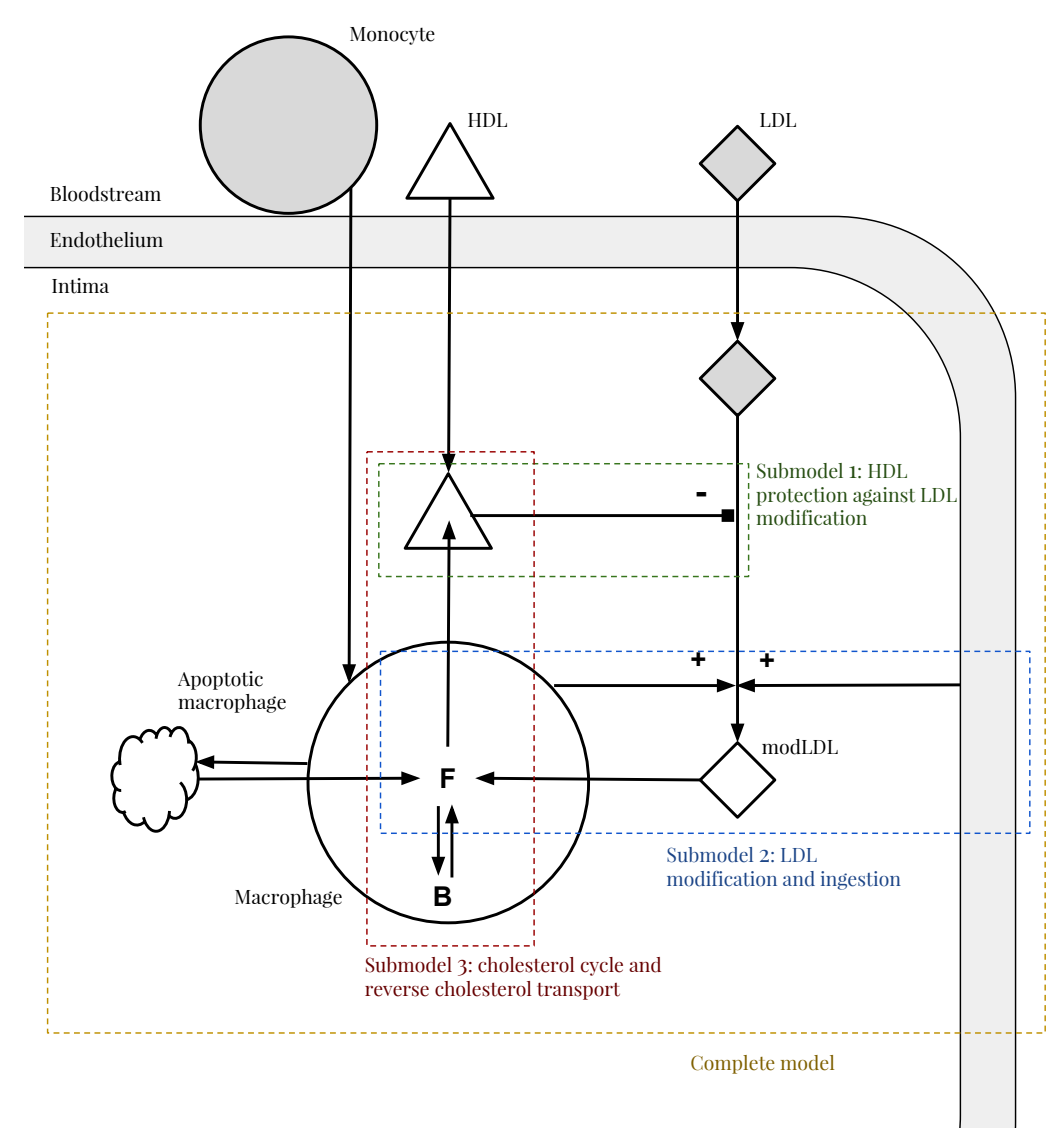

Fig. 1 Overview of the key inflammatory and lipid processes in an atherosclerotic plaque that are considered in the complete model. We assume that monocytes (circle, grey), LDL (diamond, grey) and HDL (triangle) enter the vessel wall at a constant rate which reflect their serum concentrations and the magnitude of wall shear stress exerted on the endothelium by blood flow. Monocytes differentiate into macrophages (circle, white), consume modLDL (diamond, white) and apoptotic macrophages (cloud) and gain free cholesterol (F) as a result, export cholesterol to HDL and undergo apoptosis. Free cholesterol is converted to esterified cholesterol (B) and stored in lipid droplets so free cholesterol is within an essential and cytotoxic limit. LDL is oxidatively modified by macrophages and the endothelium whereas HDL counteracts LDL oxidation. The processes considered in each submodel of in vitro systems are encircled by green (submodel 1), blue (submodel 2) and red (submodel 3) frames. The processes considered in the complete model are encircled by a yellow frame. 
is represented as a function of time only. Concentrations are either in units of mass per unit volume or number per unit volume where the representative volume will be either the volume determined by the petri dish (for in vitro experiments) or the volume of the plaque (for in vivo experiments).

Where a species in a model exists in many states, we will assume that the different states can be binned so that different classes in the model have functionally distinct roles. For example, LDL has many degrees and different types of oxidative modification 65. In our models, however, we divide LDL into two classes which we label, native LDL and modLDL. The distinction is that only modLDL creates an immune reaction that leads to inflammation. In the same way we model only those macrophages that ingest and store lipids to a significant extent, as this is the behaviour of the macrophages observed in the in vitro experiments that we use to find parameters. Macrophages in vivo exhibit a wide variety of phenotypes [48], but modeling that phenotypic diversity is beyond the capability of the available data and beyond the scope of this study.

Based on in vitro experiments and other literature we consider the following key species and their interactions in our complete model for early atherosclerotic plaques: concentration of native LDL $\ell$, concentration of modified LDL $\tilde{\ell}$, concentration of native HDL $h$, concentration of intracellular free cholesterol $f$, concentration of intracellular cholesterol ester $b$ and density of macrophages $m$. A schematic overview of the key species and their interactions is shown in Fig 1 The time-dependent behaviour of species is modeled by systems of ordinary differential equations. If specific experimental data exists we choose relationships which best represent the data. In contrast, we use linear relationships if no experimental data exists and there is nothing to indicate that the relationship must be nonlinear. The results of the mathematical submodels are least-squares fitted to various experimental measurements from literature to find values of the unknown parameters. Due to a lack of uniform data we do not distinguish between experimental results gained from cells or lipids from different animal models. We convert all quantities to SI units from the experiment-specific units used in the literature which are often non-SI units. Table 1 shows the values used for the conversions in this study.

The three submodels that we develop and validate for the in vitro experimental systems are combined to produce a complete model that describes early development of atherosclerotic plaques. The sensitivity of the complete model is analyzed with respect to the estimated parameters and critical parameters identified. The long-term behaviour of the model is analyzed which leads to a characterization of stable and unstable plaques. We usually omit the time dependencies to keep the notation clear, except in cases where it is crucial.

\subsection{Submodels of in vitro systems}

Three mathematical submodels that describe various in vitro experiments are developed. All parameters of the submodels are either experiment-specific (i.e. 
Table 1 Overview of values of key quantities in SI units.

\begin{tabular}{|c|c|c|c|}
\hline Symbol & Description & Value & Source \\
\hline$N_{\mathrm{A}}$ & Avogadro constant & $6.022 \cdot 10^{23} \frac{1}{\mathrm{Mol}}$ & \\
\hline$\rho_{1}$ & Number of cell proteins per cell volume & $3.0 \cdot 10^{15} \frac{1}{\mathrm{~mm}^{3}}$ & 43 \\
\hline$\rho_{2}$ & Molecular weight of cell proteins & $5.3 \cdot 10^{4} \frac{\mathrm{g}}{\mathrm{Mol}}$ & 39 \\
\hline $\mathrm{M}_{b}$ & Molecular weight of cholesterol ester & $6.48 \cdot 10^{2} \frac{\mathrm{Mg}}{\mathrm{Mgl}}$ & 69 \\
\hline $\mathrm{M}_{f}$ & Molecular weight of free cholesterol & $3.87 \cdot 10^{2} \frac{\mathrm{g}}{\mathrm{Mol}}$ & 69 \\
\hline$\rho_{3}$ & Molecular weight of apolipoprotein B-100 & $5.49 \cdot 10^{5} \frac{\mathrm{g}}{\mathrm{Mol}}$ & 56 \\
\hline$\rho_{4}$ & Murine (J774) macrophage volume & $2.10 \cdot 10^{-6} \mathrm{~mm}^{3}$ & 42 \\
\hline$\rho_{5}$ & Macrophages per cell protein mass & $1.80 \cdot 10^{9} \frac{1}{\mathrm{~g}}$ & $=\frac{N_{A}}{\rho_{1} \rho_{2} \rho_{4}}$ \\
\hline $\mathrm{M}_{\ell}$ & Molecular weight of LDL & $2.93 \cdot 10^{6} \frac{\mathrm{g}}{\mathrm{Mol}}$ & 1356 \\
\hline$\rho_{6}$ & Fraction of cholesterol ester of LDL mass & $38.3 \%$ & 69 \\
\hline$\rho_{7}$ & Fraction of free cholesterol of LDL mass & $8.8 \%$ & 69 \\
\hline$\rho_{8}$ & LDL particles per LDL cholesterol mass & $4.36 \cdot 10^{17} \frac{1}{\mathrm{~g}}$ & $=\frac{N_{A}}{\mathrm{M}_{0}\left(\rho_{6}+e\right.}$ \\
\hline$\rho_{9}$ & Apolipoprotein B-100 fraction of LDL protein mass & $95 \%$ & 56 \\
\hline$\rho_{10}$ & LDL particles per LDL protein mass & $1.04 \cdot 10^{18} \frac{1}{\mathrm{~g}}$ & $=\frac{N_{A} \rho_{9}}{\rho_{3}}$ \\
\hline $\mathrm{M}_{h}$ & Molecular weight of HDL & $2.92 \cdot 10^{5} \frac{g}{\mathrm{Mol}}$ & 30 \\
\hline$\rho_{11}$ & Cholesterol ester molecules per HDL particle & 110.1 & 30 \\
\hline$\rho_{12}$ & Free cholesterol molecules per HDL particle & 27.7 & 30 \\
\hline$\rho_{13}$ & HDL particles per HDL cholesterol mass & $7.34 \cdot 10^{18} \frac{1}{\mathrm{~g}}$ & $=\frac{N_{A}}{\mathrm{M}_{b} \rho_{11}+\mathrm{M}_{f} \rho_{12}}$ \\
\hline$\rho_{14}$ & HDL particles per HDL protein mass & $4.22 \cdot 10^{18} \frac{1}{\mathrm{~g}}$ & \\
\hline
\end{tabular}

All values are converted to $\mathrm{mm}, \mathrm{g}$ and $\mathrm{Mol}$.

are specified by the experimental procedure) or estimated by a least-squares fit to the measured experimental results.

\subsubsection{Submodel 1: LDL modification and ingestion}

The submodel of oxidative modification of LDL by endothelial cells and macrophages and the ingestion of native and modified LDL by macrophages is based on in vitro experiments by Henriksen et al. 27] and Leake et al. [32].

Experimental setups. The modification of LDL by endothelial cells and the ingestion of modLDL by macrophages in vitro was investigated in [27] using a two-staged experimental setup. First, specified initial concentrations of native LDL $\ell_{\text {Mod,0 }}$ were modified by exposure to cultured endothelial cell monolayers $e_{\text {Mod }}$ for specified time periods $T_{\text {Mod }}$. (Here the subscript "Mod" indicates quantities of the first stage of the experimental setup.) Subsequently, the mixtures of native and modified LDL, that had been created in the first step, were reduced to specified initial concentrations $\ell_{\text {Ing, } 0}$ and exposed to specified densities of macrophages $m_{\text {Ing }}$ for specified time periods $T_{\text {Ing }}$ to allow the macrophages to ingest the LDL. (The subscript "Ing" indicates quantities of the second stage of the experimental setup.) The ingested LDL per macrophage was measured for various experiment-specific values of $\ell_{\mathrm{Mod}, 0}, e_{\mathrm{Mod}}, T_{\mathrm{Mod}}$, $\ell_{\text {Ing, } 0}, m_{\text {Ing }}$ and $T_{\text {Ing. }}$. We also use measurements in 32. where the modification 
and ingestion of LDL by macrophages was investigated. This study used similar experimental protocols to [27] but the modification of LDL in the first stage was done using specified densities of macrophages $m_{\text {Mod }}$ instead of endothelial cell monolayers. Also, in the second stage a high concentration of foetal calf serum was added preventing the oxidative modification of LDL by macrophages. The ingested LDL per macrophage in the second stage was measured for various experiment-specific values of $\ell_{\mathrm{Mod}, 0}, m_{\mathrm{Mod}}, T_{\mathrm{Mod}}, \ell_{\mathrm{Ing}, 0}, m_{\mathrm{Ing}}$ and $T_{\mathrm{Ing}}$.

Mathematical submodel. The experimental observations in [27] and [32] are driven by the ingestion of native and modified LDL by macrophages and by the modification of native LDL by endothelial cells and macrophages. In these experiments, ingestion of native LDL, concentration $\ell$ and modified LDL, concentration $\tilde{\ell}$ per macrophage saturates as LDL concentrations increase (see [27, Fig 7 and 8 and [32, Fig 4). We describe the modification of native LDL by macrophages and endothelial cells by linear relationships in their concentrations $\ell, m$ and $e$, respectively, as no experimental data exists which indicates otherwise. We take account of the observed lag phase for the modification of native LDL of $3-8 \mathrm{~h}$ (see [27], Fig 2, [32, Fig 1a and 1b, [47, Fig 1b) by a reduction of $4 \mathrm{~h}$ of the experimental time period $T_{\mathrm{Mod}}$.

The mathematical submodel is formulated in the same units that are used in the experiments: time $[t]=\mathrm{h}$, concentration of native LDL $\left[\ell_{\mathrm{Mod}}\right]=$ $\left[\ell_{\text {Ing }}\right]=\frac{\mu \mathrm{g} \text { lipid protein }}{\mathrm{ml}}$, concentration of modified LDL $\left[\tilde{\ell}_{\mathrm{Mod}}\right]=\left[\tilde{\ell}_{\mathrm{Ing}}\right]=$ $\frac{\mu \mathrm{g} \text { lipid protein }}{\mathrm{ml}}$, concentration of total ingested LDL $\left[a_{\mathrm{Ing}}\right]=\frac{\mu \mathrm{g} \text { lipid protein }}{\mathrm{ml}}$, density of macrophages $\left[m_{\mathrm{Mod}}\right]=\left[m_{\mathrm{Ing}}\right]=\frac{\mathrm{mg} \text { cell protein }}{\mathrm{ml}}$ and density of endothelial cells $\left[e_{\mathrm{Mod}}\right]=\frac{\mathrm{mm}^{2}}{\mathrm{ml}}$. The submodel consists of two sequential system of ordinary differential equations and associated initial conditions. As in the experiments described in [27,32], the first system corresponds to the oxidative modification of LDL by macrophages and endothelial cells

$$
\begin{aligned}
& \frac{\mathrm{d}}{\mathrm{d} t} \ell_{\mathrm{Mod}}(t)=-\underbrace{\mu_{\ell} \frac{\left(\ell_{\mathrm{Mod}}\right)^{n_{\ell}}}{\left(\xi_{\ell}\right)^{n_{\ell}}+\left(\ell_{\mathrm{Mod}}\right)^{n_{\ell}}} m_{\mathrm{Mod}}}_{\begin{array}{c}
\text { ingestion of LDL } \\
\text { by macrophages }
\end{array}}-\underbrace{q_{\ell, m} \ell_{\mathrm{Mod}} m_{\mathrm{Mod}}}_{\begin{array}{c}
\text { modification of LDL } \\
\text { by macrophages }
\end{array}} \\
& -\underbrace{q_{\ell, e} \ell_{\mathrm{Mod}} e_{\mathrm{Mod}}}_{\begin{array}{c}
\text { modification of LDL } \\
\text { by endothelial cells }
\end{array}}, \\
& \frac{\mathrm{d}}{\mathrm{d} t} \tilde{\ell}_{\operatorname{Mod}}(t)=-\underbrace{\mu_{\tilde{\ell}} \frac{\left(\tilde{\ell}_{\mathrm{Mod}}\right)^{n_{\tilde{\ell}}}}{\left(\xi_{\tilde{\ell}}\right)^{n_{\tilde{\ell}}}+\left(\tilde{\ell}_{\mathrm{Mod}}\right)^{n_{\tilde{\ell}}}} m_{\mathrm{Mod}}}_{\begin{array}{c}
\text { ingestion of modLDL } \\
\text { by macrophages }
\end{array}}+\underbrace{q_{\ell, m} \ell_{\mathrm{Mod}} m_{\mathrm{Mod}}}_{\begin{array}{c}
\text { modification of LDL } \\
\text { by macrophages }
\end{array}} \\
& +\underbrace{q_{\ell, e} \ell_{\mathrm{Mod}} e_{\mathrm{Mod}}}_{\begin{array}{c}
\text { modification of LDL } \\
\text { by endothelial cells }
\end{array}}, \\
& \ell_{\text {Mod }}(4 \mathrm{~h})=\ell_{\text {Mod }, 0}, \quad \tilde{\ell}_{\operatorname{Mod}}(4 \mathrm{~h})=0, \quad t \in\left[4 \mathrm{~h} ; T_{\mathrm{Mod}}\right]
\end{aligned}
$$


and the second system to the ingestion of LDL by macrophages

$$
\begin{aligned}
& \frac{\mathrm{d}}{\mathrm{d} t} \ell_{\mathrm{Ing}}(t)=-\underbrace{\mu_{\ell} \frac{\left(\ell_{\mathrm{Ing}}\right)^{n_{\ell}}}{\left(\xi_{\ell}\right)^{n_{\ell}}+\left(\ell_{\mathrm{Ing}}\right)^{n_{\ell}}} m_{\mathrm{Ing}}}_{\begin{array}{c}
\text { ingestion of LDL } \\
\text { by macrophages }
\end{array}}-\underbrace{q_{\ell, m} \ell_{\mathrm{Ing}} m_{\mathrm{Ing}}}_{\begin{array}{c}
\text { modification of LDL } \\
\text { by macrophages }
\end{array}}, \\
& \frac{\mathrm{d}}{\mathrm{d} t} \tilde{\ell}_{\text {Ing }}(t)=-\underbrace{\mu_{\tilde{\ell}} \frac{\left(\tilde{\ell}_{\text {Ing }}\right)^{n_{\tilde{\ell}}}}{\left(\xi_{\tilde{\ell}}\right)^{n_{\tilde{\ell}}}+\left(\tilde{\ell}_{\text {Ing }}\right)^{n_{\tilde{\ell}}}} m_{\text {Ing }}}_{\begin{array}{c}
\text { ingestion of modLDL } \\
\text { by macrophages }
\end{array}}+\underbrace{q_{\ell, m} \ell_{\text {Ing }} m_{\text {Ing }}}_{\begin{array}{c}
\text { modification of LDL } \\
\text { by macrophages }
\end{array}}, \\
& \frac{\mathrm{d}}{\mathrm{d} t} a_{\text {Ing }}(t)=+\underbrace{\mu_{\ell} \frac{\left(\ell_{\text {Ing }}\right)^{n_{\ell}}}{\left(\xi_{\ell}\right)^{n_{\ell}}+\left(\ell_{\text {Ing }}\right)^{n_{\ell}}} m_{\text {Ing }}}_{\begin{array}{c}
\text { ingestion of LDL } \\
\text { by macrophages }
\end{array}}+\underbrace{\mu_{\tilde{\ell}} \frac{\left(\tilde{\ell}_{\text {Ing }}\right)^{n_{\tilde{\ell}}}}{\left(\xi_{\tilde{\ell}}\right)^{n_{\tilde{\ell}}}+\left(\tilde{\ell}_{\text {Ing }}\right)^{n_{\tilde{\ell}}}} m_{\text {Ing }}}_{\begin{array}{c}
\text { ingestion of modLDL } \\
\text { by macrophages }
\end{array}}, \\
& \ell_{\text {Ing }}(0)=\ell_{\text {Ing }, 0} \underbrace{\frac{\ell_{\text {Mod }}\left(T_{\text {Mod }}\right)}{\ell_{\text {Mod }}\left(T_{\text {Mod }}\right)+\tilde{\ell}_{\text {Mod }}\left(T_{\text {Mod }}\right)}}_{\begin{array}{c}
\text { fraction of LDL at end of } \\
\text { first experimental stage }
\end{array}}, \\
& \tilde{\ell}_{\text {Ing }}(0)=\ell_{\text {Ing, }, 0} \underbrace{\frac{\tilde{\ell}_{\text {Mod }}\left(T_{\text {Mod }}\right)}{\ell_{\text {Mod }}\left(T_{\text {Mod }}\right)+\tilde{\ell}_{\text {Mod }}\left(T_{\text {Mod }}\right)}}_{\begin{array}{c}
\text { fraction of modLDL a a end of } \\
\text { first experimental stage }
\end{array}}, \\
& a_{\text {Ing }}(0)=0, \quad t \in\left[0 ; T_{\text {Ing }}\right],
\end{aligned}
$$

where $T_{\text {Mod }}, \ell_{\text {Mod }, 0}, m_{\text {Mod }}, e_{\mathrm{Mod}}, T_{\text {Ing }}, \ell_{\text {Ing }, 0}$ and $m_{\text {Ing }}$ are experiment-specific parameters (see Appendix 1: Experiment-specific parameters of submodels, Table 5). The remaining constants $q_{\ell, m}, q_{\ell, e}, \mu_{\ell}, \xi_{\ell}, n_{\ell}, \mu_{\tilde{\ell}}, \xi_{\tilde{\ell}}$ and $n_{\tilde{\ell}}$ are the unknown parameters of the submodel. We estimate them using a least-squares fit of the simulated ingestion of native and modified LDL per macrophage in the ingestion phase $\frac{a_{\text {Ing }}\left(T_{\text {Ing }}\right)}{m_{\text {Ing }}}$ to the experimental results in [27] and [32].

\subsubsection{Submodel 2: HDL protection against LDL modification}

The submodel of HDL protection against the oxidative modification of LDL is based on in vitro experiments by Mackness et al. [41.

Experimental setup. The inhibition of oxidative modification of LDL by copper sulfate by HDL in vitro was investigated in 41. Specified initial concentrations of native LDL $\ell_{0}$ and HDL $h_{0}$ were exposed to specified concentrations of copper sulfate $\varsigma$ for specified time periods $T_{\text {Mod }}$. The lipid peroxide content per lipoprotein particle was measured for various experiment-specific values of $\ell_{0}, h_{0}, \varsigma$ and $T_{\mathrm{Mod}}$. 
Mathematical submodel. The experimental observations in 41] are driven by the modification of native LDL and native HDL by copper sulfate as well as by the inhibition of LDL modification by native HDL. The protection that HDL gives against modification of native LDL by copper sulfate saturates as the concentration of HDL $h$ increases (see 41, Fig 5). We describe the modification of native LDL and native HDL by copper sulfate by linear relationships in their concentrations $\ell, h$ and $\varsigma$, respectively, as no experimental data exists which indicates otherwise. We use the lipid peroxide quantities as a measure of the concentrations of modLDL and modified HDL. Therefore, we convert each mg of lipid protein of modified LDL and modified HDL as given in [41] to $N_{\tilde{\ell}} \mathrm{nMol}$ and $N_{\tilde{h}} \mathrm{nMol}$ of lipid peroxide, respectively.

The mathematical submodel is formulated in the following units: time $[t]=\mathrm{h}$, concentration of native LDL $[\ell]=\frac{\mathrm{mg} \text { protein }}{\mathrm{ml}}$, concentration of modified LDL $[\tilde{\ell}]=\frac{\mathrm{mg} \text { protein }}{\mathrm{ml}}$, concentration of native HDL $[h]=\frac{\mathrm{mg} \text { protein }}{\mathrm{ml}}$, concentration of modified HDL $[\tilde{h}]=\frac{\mathrm{mg} \text { protein }}{\mathrm{ml}}$ and concentration of copper sulfate $[\varsigma]=\frac{\mu \mathrm{Mol}}{\mathrm{ml}}$. The submodel consists of a system of four ordinary differential equations and associated initial conditions

$$
\begin{gathered}
\frac{\mathrm{d}}{\mathrm{d} t} \ell(t)=-\underbrace{q_{\ell, \varsigma} \ell \varsigma}_{\begin{array}{c}
\text { modification of LDL } \\
\text { by copper sulfate }
\end{array}} \cdot \underbrace{\frac{\left(K_{h}\right)^{n_{h}}}{\left(K_{h}\right)^{n_{h}}+h^{n_{h}}}}_{\begin{array}{c}
\text { inhibition of } \\
\text { modification by HDL }
\end{array}}=-\frac{\mathrm{d}}{\mathrm{d} t} \tilde{\ell}(t), \\
\frac{\mathrm{d}}{\mathrm{d} t} h(t)=-\underbrace{q_{h, \varsigma} h \varsigma}_{\begin{array}{c}
\text { modification of } \mathrm{HDL} \\
\text { by copper sulfate }
\end{array}} \cdot \underbrace{\frac{\left(K_{h}\right)^{n_{h}}}{\left(K_{h}\right)^{n_{h}}+h^{n_{h}}}}_{\begin{array}{c}
\text { modification by HDL } \\
\text { motion of }
\end{array}}=-\frac{\mathrm{d}}{\mathrm{d} t} \tilde{h}(t), \\
\ell(0)=\ell_{0}, \quad \tilde{\ell}(0)=0, \quad h(0)=h_{0}, \quad \tilde{h}(0)=0, \quad t \in\left[0 ; T_{\text {Mod }}\right],
\end{gathered}
$$

where $T_{\text {Mod }}, \ell_{0}, h_{0}$ and $\varsigma$ are experiment-specific parameters (see Appendix 1: Experiment-specific parameters of submodels Table 6). The remaining constants $N_{\tilde{\ell}}, N_{\tilde{h}}, q_{\ell, \varsigma}, q_{h, \varsigma}, K_{h}$ and $n_{h}$ are the unknown parameters of the submodel. We estimate them using a least-squares fit of the simulated lipid peroxide content per lipoprotein $\frac{N_{\tilde{\ell}} \tilde{\ell}\left(T_{\mathrm{Mod}}\right)}{\ell_{0}}$ and the reduction of lipid peroxide content due to the presence of HDL to the experimental results in [41.

\subsubsection{Submodel 3: cholesterol cycle and reverse cholesterol transport}

Macrophages ingest LDL and store its cholesterol content as intracellular free and esterified cholesterol. Free and esterified cholesterol are in a dynamic equilibrium due to hydrolysis and esterification which together are referred to as cholesterol cycle. Further, macrophages are able to offload their free cholesterol content on to native HDL, a process called reverse cholesterol transport. The submodel of cholesterol cycle and reverse cholesterol transport is based on in vitro experiments by Brown et al. [4,5]. 
Experimental setup. The ingestion of cholesterol by macrophages and the cholesterol cycle within macrophages in vitro was investigated in [4. Specified concentrations of modified LDL $\tilde{\ell}_{0}$ were incubated in a dish in the presence of specified densities of macrophages $m$ for specified time periods $T_{\text {Cho }}$. The intracellular free cholesterol and cholesterol ester per macrophage were measured for various experiment-specific values of $\tilde{\ell}_{0}, m$ and $T_{\text {Cho }}$.

Using this study [4] as a basis, the cholesterol efflux from macrophages to HDL was investigated in [5] using a multi-staged experimental setup. First, specified densities of macrophages $m$ were loaded with free and esterified cholesterol by incubation with LDL. Subsequently, the concentrations of intracellular free cholesterol $f_{0}$ and esterified cholesterol $b_{0}$ per macrophage were measured. After specified time periods $T_{h}$, specified concentrations of HDL $h_{0}$ were added. After a total time period of $T_{\text {Cho }}$ the intracellular free and esterified cholesterol per macrophage as well as the excreted cholesterol per macrophage was measured for various experiment-specific values of $m, f_{0}, b_{0}, h_{0}, T_{h}$ and $T_{\text {Cho }}$. In both studies, the modification of LDL by macrophages was prevented by high concentrations of foetal calf serum.

Mathematical submodel. The experimental observations in [4] and [5] are driven by the ingestion of modLDL by macrophages, the free cholesterol-cholesterol ester cycle within macrophages and the offloading of free cholesterol from macrophages to HDL. The ingestion of modLDL per macrophage saturates as the concentration of modLDL $\tilde{\ell}$ increases (see [27, Fig 7). Each $\mu \mathrm{g}$ lipid protein of ingested modLDL can be identified with $N_{f}$ nMol of incorporated intracellular cholesterol particles which are hydrolyzed to free cholesterol [5]. The cholesterol cycle of free cholesterol and cholesterol ester within macrophages shows a buffer-like behaviour (see [4], Fig 1 and [5], Fig 1 and 4). Hence, we assume that there exists a concentration of free cholesterol $f_{\text {Min }}$ which is favored by macrophages as well as a maximum concentration of free cholesterol $f_{\mathrm{Max}}$ possible within macrophages [5]. As suggested in [5], these concentrations $f_{\text {Min }}$ and $f_{\mathrm{Max}}$ affect only the rate $k_{f}$ of esterification and not the rate $k_{b}$ of hydrolysis. The delivery of free cholesterol from macrophages to native HDL saturates as the concentration of HDL $h$ increases (see [5], Fig 2), but only takes place when concentration of intracellular free cholesterol $f$ is bigger than $f_{\text {Min }}$ (see [5, Fig 1b). In accordance with the results in [5], we assume that native HDL is never saturated by the cholesterol it takes up and so the action of HDL does not decline through lipid loading due to reverse cholesterol transport. Additionally, a constant efflux of intracellular free cholesterol from macrophages independent of native HDL (see 4], Fig 1a and [5], Fig 1c) occurs in vitro.

The mathematical submodel is formulated in the following units: time $[t]=\mathrm{h}$, concentration of modified LDL $\left[\tilde{\ell}_{0}\right]=\frac{\mu \mathrm{g} \text { lipid protein }}{\mathrm{ml}}$, concentration of native HDL $[h]=\frac{\mu \mathrm{g} \text { lipid protein }}{\mathrm{ml}}$, density of macrophages $[\mathrm{m}]=\frac{\mathrm{mg} \text { cell protein }}{\mathrm{ml}}$, concentration of total intracellular free cholesterol $[f]=\frac{\mathrm{nMol}}{\mathrm{ml}}$, concentration of total intracellular cholesterol ester $[b]=\frac{\mathrm{nMol}}{\mathrm{ml}}$ and concentration of total 
cholesterol excreted from cells $[r]=\frac{\mathrm{nMol}}{\mathrm{ml}}$. The submodel consists of a system of three ordinary differential equations and associated initial conditions

$$
\begin{aligned}
& h(t)=h_{0} \mathcal{H}\left(t-T_{h}\right) \\
& \frac{\mathrm{d}}{\mathrm{d} t} f(t)=+N_{f} \underbrace{\mu_{\tilde{\ell}} \frac{\left(\tilde{\ell}_{0}\right)^{n_{\tilde{\ell}}}}{\left(\xi_{\tilde{\ell}}\right)^{n_{\tilde{\ell}}}+\left(\tilde{\ell}_{0}\right)^{n_{\tilde{\ell}}}} m}_{\text {ingestion of modLDL }}-\underbrace{k_{f} \frac{\frac{f}{m}-f_{\text {Min }}}{f_{\text {Max }}-\frac{f}{m}}\left(\frac{f}{m}-f_{\text {Min }}\right) m}_{\text {esterification of free cholesterol }} \\
& +\underbrace{k_{b} b}_{\begin{array}{c}
\text { hydrolysis of } \\
\text { cholesterol ester }
\end{array}}-\underbrace{\left(c_{f}+\mu_{f} \frac{h^{n_{f}}}{\left(\xi_{f}\right)^{n_{f}}+h^{n_{f}}}\right)\left(\frac{f}{m}-f_{\text {Min }}\right) m}_{\text {cholesterol efflux from macrophages }}, \\
& \frac{\mathrm{d}}{\mathrm{d} t} b(t)=+\underbrace{k_{f} \frac{\frac{f}{m}-f_{\text {Min }}}{f_{\text {Max }}-\frac{f}{m}}\left(\frac{f}{m}-f_{\text {Min }}\right) m}_{\text {esterification of free cholesterol }}-\underbrace{k_{b} b}_{\begin{array}{c}
\text { hydrolysis of } \\
\text { cholesterol ester }
\end{array}}, \\
& \frac{\mathrm{d}}{\mathrm{d} t} r(t)=+\underbrace{\left(c_{f}+\mu_{f} \frac{h^{n_{f}}}{\left(\xi_{f}\right)^{n_{f}}+h^{n_{f}}}\right)\left(\frac{f}{m}-f_{\text {Min }}\right) m}_{\text {cholesterol efflux from macrophages }}, \\
& f(0)=f_{0} m, \quad b(0)=b_{0} m, \quad r(0)=0, \quad t \in\left[0 ; T_{\text {Cho }}\right],
\end{aligned}
$$

where the function $\mathcal{H}$ denotes the Heavyside step function and $T_{\text {Cho }}, T_{h}, h_{0}, \tilde{\ell}_{0}$, $f_{0}, b_{0}$ and $m$, are experiment-specific parameters (see Appendix 1: Experimentspecific parameters of submodels. Table 7). The constants $\mu_{\tilde{\ell}}, \xi_{\tilde{\ell}}, n_{\tilde{\ell}}$ are the same as those introduced in submodel 1 . We use the values of these parameters determined by the fitting of submodel 1 , see Table 4 . The remaining constants $N_{f}, f_{\mathrm{Min}}, f_{\mathrm{Max}}, k_{f}, k_{b}, c_{f}, \mu_{f}, \xi_{f}$ and $n_{f}$ are the unknown parameters of submodel 3 and are estimated using a least-squares fit of the simulated concentration of intracellular free cholesterol $\frac{f\left(T_{\text {Cho }}\right)}{m}$, intracellular cholesterol ester $\frac{b\left(T_{\text {Cho }}\right)}{m}$ and excreted cholesterol $\frac{r\left(T_{\text {Cho }}\right)}{m}$ per macrophage to the experimental results in [4] and [5]. The results in [4] are convert into the correct units for the submodel using the molecular weights of free cholesterol $\mathrm{M}_{f}=3.87 \cdot 10^{2} \frac{\mathrm{g}}{\mathrm{Mol}}$ and cholesterol ester $\mathrm{M}_{b}=6.48 \cdot 10^{2} \frac{\mathrm{g}}{\text { Mol }}[69$. Additionally, we use doubled results of [5], Fig 2 in order to be consistent with other measurements of the same experiment, i.e. [5], Fig 1c, 4 and 7b.

\subsection{Complete model of early atherosclerotic plaques}

The purpose of the complete mathematical model is to predict the development of early stages of atherosclerotic plaques as they may occur in vivo. It is based on the previous submodels of in vitro systems that quantified the modification and ingestion of LDL by macrophages and endothelial cells, the protective action of HDL against LDL modification, the cholesterol cycle within macrophages and reverse cholesterol transport from macrophages. Hence, we assume that 
the previous assumptions underlying the submodels and parameters of the in vitro systems are valid in an in vivo setting too. This modeling approach is philosophically the same as by experimental scientists and relies on the idea that results from in vitro studies can be used to inform our understanding of what goes on in vivo.

We assume that the protection against LDL modification that HDL provides is independent of the source of modification, as no experimental data exists which indicates otherwise. Hence, we assume that the protection HDL gives against oxidative modification of LDL by copper is also valid for endothelial cell and macrophage mediated modification. Following [11] the rates of modification of HDL by macrophages and endothelial cells are 7.6 times smaller than the rates that LDL is modified by macrophages and endothelial cells. Modified HDL no longer takes part in the reverse cholesterol transport 49.

In contrast to the in vitro systems where the experiments were run in hours or days, the disease progression in vivo is on a much longer time scale. Hence, the fate of macrophages in vivo is of particular interest. Macrophages can undergo necrosis, proliferation, egress and apoptosis. But as rates of necrosis, proliferation and egress are small in early stages of atherosclerosis (see [38, 54,57), the only fate of macrophages considered in the model is apoptosis. The rate of macrophage apoptosis is dependent on the concentration of free cholesterol within macrophages (see [77, Fig 4a), which we approximate by the average concentration $\frac{f}{m}$ of intracellular free cholesterol per macrophage. As it is implausible to assume an unlimited rate of macrophages apoptosis and it is in accordance with the results in [77, we assume that the rate of apoptosis saturates (with an exponent of $n_{m}=2$ ) as the concentration of free cholesterol $f$ increases. In [77] the initial population of macrophages $m(t)$ decays exponentially over the time period of the experiment $T_{\mathrm{Apo}}=9 \mathrm{~h}$. This gives:

$$
m\left(T_{\mathrm{Apo}}\right)=m_{0} \exp \left(-\mu_{m} \frac{\left(\frac{f_{0}}{m_{0}}\right)^{n_{m}}}{\left(\xi_{m}\right)^{n_{m}}+\left(\frac{f_{0}}{m_{0}}\right)^{n_{m}}} T_{\mathrm{Apo}}\right),
$$

where $\frac{f_{0}}{m_{0}}$ is the experiment-specific average intracellular free cholesterol of the macrophage population. The unknown parameters $\mu_{m}$ and $\xi_{m}$ are least-squares fitted to the measurements of Yao et al. [77], Fig 4a. The total intracellular free and esterified cholesterol is not affected by apoptosis as we assume that all apoptotic macrophages are reingested by other non-apoptotic macrophages by efferocytosis.

The concentrations of native LDL $\ell$ and native HDL $h$ and the density of macrophages $m$ in a plaque are determined by recruitment from the bloodstream. Hence, rates of recruitment of native LDL $r_{\ell}$, of native HDL $r_{h}$ and of macrophages $r_{m}$ (per unit area of plaque surface) are introduced into the complete model. Monocytes in the blood contain a concentration of free cholesterol $f_{\text {In }}$ [77] that remains inside each monocyte-derived macrophage when it is recruited to the plaque. 
The radial thickness of the plaques is denoted by $H$. We use $H=10 \mu \mathrm{m}$ which is the width of the intima [55, 76] as we consider early stages of atherosclerosis. The factor $\frac{1}{H}$ is introduced to account for effects of processes which take place on plaque surface (such as the inward flux of lipids and cells from the blood and the modification of LDL and HDL by endothelial cells) on volume-averaged concentrations.

Mathematical model. The complete model is formulated in the following units: time $[t]=\mathrm{h}$, concentration of native LDL $[\ell]=\frac{1}{\mathrm{~mm}^{3}}$, concentration of modified LDL $[\tilde{\ell}]=\frac{1}{\mathrm{~mm}^{3}}$, concentration of native HDL $[h]=\frac{1}{\mathrm{~mm}^{3}}$, concentration of total intracellular free cholesterol $[f]=\frac{1}{\mathrm{~mm}^{3}}$, concentration of total intracellular cholesterol ester $[b]=\frac{1}{\mathrm{~mm}^{3}}$ and density of macrophages $[m]=\frac{1}{\mathrm{~mm}^{3}}$. The complete model consist of a system of six ordinary differential equations and associated initial conditions

$$
\begin{aligned}
& \frac{\mathrm{d}}{\mathrm{d} t} \ell(t)=-\underbrace{\mu_{\ell} \frac{\ell^{n_{\ell}}}{\left(\xi_{\ell}\right)^{n_{\ell}}+\ell^{n_{\ell}}} m}_{\begin{array}{c}
\text { ingestion of LDL } \\
\text { by macrophages }
\end{array}}-\underbrace{\left(q_{\ell, m} \ell m+\frac{q_{\ell, e}}{H} \ell\right)}_{\begin{array}{c}
\text { modification of LDL by } \\
\text { macrophages and } \\
\text { endothelial cells }
\end{array}} \cdot \underbrace{\frac{\left(K_{h}\right)^{n_{h}}}{\left(K_{h}\right)^{n_{h}}+h^{n_{h}}}}_{\begin{array}{c}
\text { inhibition of } \\
\text { modification by HDL }
\end{array}} \\
& +\underbrace{\frac{r_{\ell}}{H}}_{\substack{\text { recruitment } \\
\text { of LDL }}} \text {, } \\
& \frac{\mathrm{d}}{\mathrm{d} t} \tilde{\ell}(t)=-\underbrace{\mu_{\tilde{\ell}} \frac{\tilde{\ell}^{n_{\tilde{\ell}}}}{\left(\xi_{\tilde{\ell}}\right)^{n_{\tilde{\ell}}}+\tilde{\ell}_{\tilde{\ell}}} m}_{\begin{array}{c}
\text { ingestion of modLDL } \\
\text { by macrophages }
\end{array}}+\underbrace{\left(q_{\ell, m} \ell m+\frac{q_{\ell, e}}{H} \ell\right)}_{\begin{array}{c}
\text { modification of LDL by } \\
\text { macrophages and } \\
\text { endothelial cells }
\end{array}} \cdot \underbrace{\frac{\left(K_{h}\right)^{n_{h}}}{\left(K_{h}\right)^{n_{h}}+h^{n_{h}}}}_{\begin{array}{c}
\text { inhibition of } \\
\text { modification by HDL }
\end{array}}, \\
& \frac{\mathrm{d}}{\mathrm{d} t} h(t)=-\underbrace{q_{h, m} h m}_{\begin{array}{c}
\text { modification of HDL } \\
\text { by macrophages }
\end{array}}-\underbrace{\frac{q_{h, e}}{H} h}_{\begin{array}{c}
\text { modification of } \\
\text { by endothelial cells }
\end{array}}+\underbrace{\frac{r_{h}}{H}}_{\begin{array}{c}
\text { recruitment } \\
\text { of HDL }
\end{array}}, \\
& \frac{\mathrm{d}}{\mathrm{d} t} f(t)=+N_{f} \underbrace{\mu_{\ell} \frac{\ell^{n_{\ell}}}{\left(\xi_{\ell}\right)^{n_{\ell}}+\ell^{n_{\ell}}} m}_{\begin{array}{c}
\text { ingestion of LDL } \\
\text { by macrophages }
\end{array}}+N_{f} \underbrace{\mu_{\tilde{\ell}} \frac{\tilde{\ell}^{n_{\tilde{\ell}}}}{\left(\xi_{\tilde{\ell}}\right)^{n_{\tilde{\ell}}}+\tilde{\ell}^{n_{\tilde{\ell}}}} m}_{\begin{array}{c}
\text { ingestion of modLDL } \\
\text { by macrophages }
\end{array}}-\underbrace{k_{f} \frac{\left(f-f_{\operatorname{Min}} m\right)^{2}}{f_{\operatorname{Max}} m-f}}_{\begin{array}{c}
\text { esterification of } \\
\text { free cholesterol }
\end{array}} \\
& +\underbrace{k_{b} b}_{\begin{array}{c}
\text { hydrolysis of } \\
\text { cholesterol ester }
\end{array}}-\underbrace{\mu_{f} \frac{h^{n_{f}}}{\left(\xi_{f}\right)^{n_{f}}+h^{n_{f}}}\left(f-f_{\mathrm{Min}} m\right)}_{\text {cholesterol efflux from macrophages }}+\underbrace{\frac{r_{m}}{H} f_{\mathrm{In}}}_{\begin{array}{c}
\text { recruitment of } \\
\text { macrophages }
\end{array}}, \\
& \frac{\mathrm{d}}{\mathrm{d} t} b(t)=+\underbrace{k_{f} \frac{\left(f-f_{\mathrm{Min}} m\right)^{2}}{f_{\mathrm{Max}} m-f}}_{\begin{array}{c}
\text { esterification of } \\
\text { free cholesterol }
\end{array}}-\underbrace{k_{b} b}_{\begin{array}{c}
\text { hydrolysis of } \\
\text { cholesterol ester }
\end{array}},
\end{aligned}
$$




$$
\begin{aligned}
\frac{\mathrm{d}}{\mathrm{d} t} m(t) & =-\underbrace{\mu_{m} \frac{f^{n_{m}}}{\left(\xi_{m} m\right)^{n_{m}}+f^{n_{m}}} m}_{\text {apoptosis of macrophages }}+\underbrace{\frac{r_{m}}{H}}_{\begin{array}{c}
\text { recruitment of } \\
\text { macrophages }
\end{array}}, \\
\ell(0) & =0, \quad \tilde{\ell}(0)=0, \quad h(0)=0, \quad f(0)=f_{0} m_{0}, \\
b(0) & =b_{0} m_{0}, \quad m(0)=m_{0}, \quad t \in[0 ; \infty[,
\end{aligned}
$$

where all parameters except the initial conditions $f_{0}, b_{0}, m_{0}$ and the rates of recruitment $r_{\ell}, r_{h}, r_{m}$ have been introduced previously.

The initial conditions describe the normal, un-inflamed state of the artery wall determined by the prior condition of the subject. We assume that the initial intracellular free cholesterol $f_{0}$ per macrophage is $50 \%$ higher than the free cholesterol $f_{\text {In }}$ of recruited macrophages. Assuming that macrophages and intracellular cholesterols are in a steady-state, we get

$$
\begin{aligned}
f_{0} & =1.5 f_{\text {In }} \\
m_{0} & =\frac{r_{m}}{H} \frac{\left(\xi_{m}\right)^{n_{m}}+\left(f_{0}\right)^{n_{m}}}{\mu_{m}\left(f_{0}\right)^{n_{m}}} \\
b_{0} & =\frac{k_{f}}{k_{b}} \frac{\left(f_{0}-f_{\mathrm{Min}}\right)^{2}}{f_{\mathrm{Max}}-f_{0}} .
\end{aligned}
$$

We set the initial conditions for the concentrations of lipids to zero as the lipids rapidly reach a non-zero equilibrium. It is important to note that the long-term behaviour of the complete model is independent of the specific choice of initial conditions.

The rates of recruitment of LDL $r_{\ell}$, of HDL $r_{h}$ and of macrophages $r_{m}$ into the plaque in reality are strongly dependent on the particular plaque under consideration, including the wall shear stress, where the plaque forms, and the diet, lifestyle and physiology of the individual who carries the plaque. In a healthy subject we estimated, that the recruitment rates can vary by an order

\begin{tabular}{|c|c|c|c|}
\hline Parameter & Description & Range & Source \\
\hline$r_{\ell}$ & Rate of LDL influx & $3 \cdot 10^{6}-3 \cdot 10^{9}{\frac{1}{\mathrm{hmm^{2 }}}}^{*} *$ & \begin{tabular}{|l|l|l|l|}
70 & 55 & 26 & 71 \\
\end{tabular} \\
\hline$r_{h}$ & Rate of HDL influx & $4 \cdot 10^{7}-4 \cdot 10^{9} \frac{1}{h-m^{2}} *$ & \begin{tabular}{l|l|l|l|}
70 & 55 & 26 & 64 \\
\end{tabular} \\
\hline$r_{m}$ & Rate of macrophage recruitment & $400-2800 \frac{1}{\mathrm{~h} \mathrm{~mm}^{2}}$ & 290 \\
\hline
\end{tabular}
of up to 100 [70,55, 26]. However, in LDL receptor deficient mice this order can even increase up to 1000 due to the very high cholesterol concentrations in the blood [71. The ranges of these rates of recruitment are given in Table 2

Table 2 Ranges of the rates of recruitment for the complete mathematical model.

Parameters indicated by ${ }^{*}$ are estimated using $\rho_{8}$ and $\rho_{13}$ from Table 1 All values are given in the units of the complete mathematical model. 
Remark. The mathematical submodels that describe the in vitro systems are special cases of the complete model of early atherosclerotic plaque development.

Remark. To keep the notation comprehensible, the symbols $f$ and $b$ have the following definitions: Stand-alone $f$ and $b$ represent (time dependent) total concentrations of intracellular free and esterified cholesterol per volume, respectively. However, with an index such as $f_{0}, f_{\text {Min }}, f_{\text {Max }}, f_{\text {In }}$ and $b_{0}$ they denote (constant) quantities of unit intracellular cholesterol per macrophage.

\subsection{Sensitivity analysis of complete model}

Most of the parameters of the complete model are estimated by least-squares fits of the previous submodels to the respective in vitro experiments. The estimated parameters have a degree of uncertainty due to the different experimental setups and measurement methods, different animals used as experimental models and measurement errors. Additionally, the conversion from the various units of measurement in the experimental studies (especially "mass of cell protein" to "amount of cells" via $\rho_{5}$ from Table 1) to a unified SI unit system introduces another source of uncertainty for some parameters.

To quantify the effect of uncertainties in the parameters on the results of the complete model, we perform a local sensitivity analysis in terms of a metabolic control analysis [79. Therefore, we compare the normalized partial derivatives of all concentrations with respect to all parameters $p$ of the complete model (see Tables 2 and 4 ) at time $T_{\mathrm{AC}}=25$ weeks. As in [79], we estimate the partial derivatives by using forward finite difference approximations with a sufficiently small variation parameter $\varepsilon=0.1 \%$. Hence, we compute the metabolic control coefficient $\operatorname{MCC}(\ell, p)$ of LDL $\ell$ with respect to the model parameter $p$ by

$$
\operatorname{MCC}(\ell, p)=\frac{1}{\ell_{p}\left(T_{\mathrm{AC}}\right)} \frac{\partial}{\partial p} \ell_{p}\left(T_{\mathrm{AC}}\right) \approx \frac{1}{\ell_{p}\left(T_{\mathrm{AC}}\right)} \frac{\ell_{p+\varepsilon \%}\left(T_{\mathrm{AC}}\right)-\ell_{p}\left(T_{\mathrm{AC}}\right)}{\varepsilon},
$$

where $\ell_{p+\varepsilon \%}$ denotes the concentration of LDL $\ell$ computed with the parameter $p$ perturbed by $\varepsilon$ percent. The metabolic control coefficients of all other species are computed in the same way. As basis for the perturbations of the recruitment rates we used $r_{\ell}=3 \cdot 10^{7} \frac{1}{\mathrm{~h} \mathrm{~mm}^{2}}, r_{h}=4 \cdot 10^{8} \frac{1}{\mathrm{~h} \mathrm{~mm}^{2}}$ and $r_{m}=1000 \frac{1}{\mathrm{~h} \mathrm{~mm}^{2}}$. For all other parameters we utilized the values stated in Table 4

\subsection{Stability analysis of complete model}

The long-term outcome for the plaque of the complete model is strongly dependent on the three recruitment rates of LDL $r_{\ell}$, of HDL $r_{h}$ and of macrophages $r_{m}$ which characterize each individual plaque. They vary by an order of up to 1000 (see Table 2) resulting in qualitatively different predicted developments of the model plaque. Hence, we focus on these three recruitment rates and perform a 
systematic analysis of the long-term stability of the complete model. Therefore, we use the following proposition:

Proposition 1. Let $\ell(t), \tilde{\ell}(t), h(t), f(t), b(t), m(t)(t \geq 0)$ be the unique and smooth solution of the initial value problem defined by equations (6) and (7) with strictly positive parameters. If $f_{\text {Min }} \leq f_{\text {In }}<f_{\text {Max }}$ and $f_{\text {Min }} \leq f_{0}<f_{\text {Max }}$, then the solution satisfies:

1. $\ell(t), \tilde{\ell}(t), h(t), f(t), b(t), m(t) \geq 0 \forall t \geq 0$.

2. $m(t) \in[\underbrace{\frac{r_{m}}{H} \frac{\left(\xi_{m}\right)^{n_{m}}+\left(f_{\text {Max }}\right)^{n_{m}}}{\mu_{m}\left(f_{\text {Max }}\right)^{n_{m}}}}_{=: m_{\text {Min }}}, \underbrace{\frac{r_{m}}{H} \frac{\left(\xi_{m}\right)^{n_{m}}+\left(f_{\text {Min }}\right)^{n_{m}}}{\mu_{m}\left(f_{\text {Min }}\right)^{n_{m}}}}_{=: m_{\text {Max }}}]$

and $\frac{f(t)}{m(t)} \in\left[f_{\text {Min }}, f_{\text {Max }}\right] \forall t \geq 0$.

3. $\ell(t), h(t), f(t), m(t)$ are bounded. If additionally $\frac{r_{\ell}}{H}<\mu_{\tilde{\ell}} m_{\mathrm{Min}}$, then $\tilde{\ell}(t)$ is bounded too.

Proof. See Appendix 3: Proof of Proposition 1.

The long-term outcome of the complete model is analyzed by numerically determining the steady-state solutions for the concentrations of macrophages $\hat{m} \in$ $\left[m_{\mathrm{Min}} ; m_{\mathrm{Max}}\right]$ and total intracellular free cholesterol per macrophage $\frac{\hat{f}}{\hat{m}} \in$ $\left[f_{\mathrm{Min}} ; f_{\mathrm{Max}}\right]$ for the full physiological spectrum of rates of recruitment $r_{\ell}, r_{h}$ and $r_{m}$ (see Table 2). We also investigate numerically the boundedness of the concentration of the total intracellular cholesterol ester $b(t)$ with respect to the recruitment rates in order to asses the severity and risk of plaques.

\section{Results}

All numerical computations were performed using a commercial software package (MATLAB 9.0, The MathWorks Inc., Natick, Massachusetts, USA, 2000).

\subsection{Model parameters}

The mathematical submodels of LDL modification and ingestion, HDL protection against LDL modification, cholesterol cycle and reverse cholesterol transport and macrophage apoptosis are numerically solved and their parameters least-squares fitted to the respective experimental results. An overview of the sources of these studies, the number of different experiments in each study and the number of data points from each study that were used in the least-squares fitting of the unknown parameters is given in Table 3 The fitted parameters and the remaining parameters of the complete model are given in Table 4. The least-squares fits of the mathematical submodels of in vitro systems to the experimental results are given in Appendix 2: Least-squares fits of submodels 
Table 3 Sources of the experimental studies, number of different experiments in each study and number of data points from each study that were used in the least-squares fitting of the mathematical submodels of in vitro systems.

\begin{tabular}{|c||c|c|}
\hline Source & Experimental setups & Number of data points \\
\hline 27 & 3 & 34 \\
{$[32$} & 2 & 24 \\
\hline 41 & 2 & 27 \\
\hline 4 & 2 & 20 \\
{$[5]$} & 6 & 65 \\
{$[7]$} & 1 & 2 \\
\hline Total & 16 & 172 \\
\hline
\end{tabular}

\subsection{Analysis of complete model}

The local sensitivity analysis of the complete mathematical model is analyzed as described in the Models and Methods section. Hence, the results of the performed metabolic control analysis are independent of the chosen variation parameter $\varepsilon=0.1 \%$. The metabolic control coefficients for LDL, modLDL, HDL, average intracellular free and esterified cholesterol per macrophage and macrophages with respect to all model parameters of the complete model are visualized in Fig 2

Data from the literature $[70,55,26,64,29,1$, suggest that the three rates of recruitment $r_{\ell}, r_{h}, r_{m}$ which characterize each individual plaque vary by an order of up to 1000 (see Table 2) and induce qualitatively different longterm outcomes of the complete model. Time-dependent concentrations for three example sets of recruitment rates with qualitatively different long-term outcomes are plotted in Fig 3

The analysis of the long-term outcome of the complete model with respect to the recruitment rates is performed as described in the Models and Methods section. Proposition 1 applies as all its requirements are fulfilled by the complete model with the parameter set from Table 4 . Hence, the steady-state concentrations of macrophages $\hat{m}$ and intracellular free cholesterol per macrophage $\frac{\hat{f}}{\hat{m}}$ satisfy

$$
\begin{aligned}
& \hat{m} \in\left[m_{\mathrm{Min}}, m_{\mathrm{Max}}\right]=\left[1.16 \cdot 10^{7}, 5.90 \cdot 10^{9}\right], \\
& \frac{\hat{f}}{\hat{m}} \in\left[f_{\mathrm{Min}}, f_{\mathrm{Max}}\right]=\left[1.22 \cdot 10^{10}, 7.15 \cdot 10^{10}\right]
\end{aligned}
$$

and only the concentration of cholesterol ester $b$ can be unbounded. Hence, the boundedness of intracellular cholesterol ester $b$, the steady-state concentration of intracellular free cholesterol per macrophages $\frac{\hat{f}}{\hat{m}}$ as well as the steadystate density of macrophages $\hat{m}$ is computed for the full physiological ranges of the rates of recruitment of LDL $r_{\ell}$, HDL $r_{h}$ and macrophages $r_{m}$. The range of recruitment rates that we consider is $r_{\ell} \in\left[3 \cdot 10^{6}, 3 \cdot 10^{9}\right] \frac{1}{\mathrm{~h} \mathrm{~mm}^{2}}$, $r_{h} \in\left[0,5 \cdot 10^{9}\right] \frac{1}{\mathrm{~h} \mathrm{~mm}^{2}}$ and $r_{m} \in[200,3000] \frac{1}{\mathrm{~h} \mathrm{~mm}^{2}}$ (compare Table 2]. It is 
Table 4 Fitted and estimated parameters of the mathematical submodels given in units of the submodel and SI units.

\begin{tabular}{|c|c|c|c|c|}
\hline Parameter & Description & $\begin{array}{l}\text { Value in units } \\
\text { of the submodels }\end{array}$ & $\begin{array}{l}\text { Value in } \\
\text { SI units }\end{array}$ & $\begin{array}{c}\text { Fitted to/ } \\
\text { Source }\end{array}$ \\
\hline$q_{\ell, m}$ & Rate of modification of $\ell$ by $m$ & $1.18 \cdot 10^{-1} \frac{\mathrm{ml}}{\mathrm{h}(\mathrm{mg} \text { cell protein })}$ & $6.56 \cdot 10^{-5} \frac{\mathrm{mm}^{3}}{\mathrm{~h}}$ & \begin{tabular}{|l|l|}
27 & 32 \\
\end{tabular} \\
\hline$q_{\ell, e}$ & Rate of modification of $\ell$ by $e$ & $2.48 \cdot 10^{-5} \frac{\mathrm{ml}^{2}}{\mathrm{h.mm}}$ & $2.48 \cdot 10^{-2} \frac{\mathrm{mm}}{\mathrm{h}}$ & \begin{tabular}{|l|l|}
27 & 32 \\
\end{tabular} \\
\hline$\mu_{\ell}$ & Rate of ingestion of $\ell$ by $m$ & $1.46 \cdot 10^{-1} \frac{\mu \mathrm{g} \text { lipid protein }}{\mathrm{h}(\mathrm{mg} \text { cell protein })}$ & $8.44 \cdot 10^{4} \frac{1}{\mathrm{~h}}$ & 2732 \\
\hline$\xi_{\ell}$ & Saturation of ingestion of $\ell$ by $m$ & $5.73 \frac{\mu \mathrm{g} \text { lipid protein }}{\mathrm{ml}}$ & $5.96 \cdot 10^{9} \frac{1}{\mathrm{~mm}^{3}}$ & 2732 \\
\hline$n_{\ell}$ & Exponent of ingestion of $\ell$ by $m$ & 1.99 & 1.99 & 2732 \\
\hline$\mu_{\tilde{\ell}}$ & Rate of ingestion of $\tilde{\ell}$ by $m$ & $7.08 \cdot 10^{-1} \frac{\mu \mathrm{g} \text { lipid protein }}{\mathrm{h}(\mathrm{mg} \text { cell protein })}$ & $4.09 \cdot 10^{5} \frac{1}{h}$ & \begin{tabular}{|l|l|}
27 & 32 \\
\end{tabular} \\
\hline$\xi_{\tilde{\ell}}$ & Saturation of ingestion of $\tilde{\tilde{\ell}}$ by $m$ & $4.63 \frac{\mu \mathrm{g} \text { lipid protein }}{\mathrm{ml}}$ & $4.82 \cdot 10^{9} \frac{1}{\mathrm{~mm}^{3}}$ & 2732 \\
\hline$n_{\tilde{\ell}}$ & Exponent of ingestion of $\tilde{\ell}$ by $m$ & 1.99 & 1.99 & \begin{tabular}{|l|l|}
27 & 32 \\
\end{tabular} \\
\hline$N_{\tilde{\ell}}$ & Lipid peroxide per $\tilde{\ell}$ & $298.49 \frac{\text { nMol }}{\text { mg lipid protein }}$ & $1.73 \cdot 10^{2}$ & 41 \\
\hline$N_{\tilde{h}}$ & Lipid peroxide per $\tilde{h}$ & $63.73 \frac{\mathrm{nMol}}{\mathrm{mg} \text { lipid protein }}$ & 9.09 & 41 \\
\hline$q_{\ell, \varsigma}$ & Rate of lipid peroxidation in $\ell$ by $\varsigma$ & $1.11 \cdot 10^{-2} \frac{\mathrm{ml}}{\mathrm{h} \mu \mathrm{Mol}}$ & $1.84 \cdot 10^{-17} \frac{\mathrm{mm}^{3}}{\mathrm{~h}}$ & 41 \\
\hline$q_{h, \varsigma}$ & Rate of lipid peroxidation in $h$ by $\varsigma$ & $6.18 \cdot 10^{-2} \frac{\mathrm{ml}}{\mathrm{h} \mu \mathrm{Mol}}$ & $1.03 \cdot 10^{-16} \frac{\mathrm{mm}^{3}}{\mathrm{~h}}$ & 41 \\
\hline$K_{h}$ & Saturation of inhibition of modification by $h$ & $2.57 \cdot 10^{-1} \frac{\mathrm{mg} \text { lipid protein }}{\mathrm{ml}}$ & $1.08 \cdot 10^{12} \frac{1}{\mathrm{~mm}^{3}}$ & 41 \\
\hline$n_{h}$ & Exponent of inhibition of modification by $h$ & 2.59 & 2.59 & 41 \\
\hline$N_{f}$ & Number of ingested $f$ per ingested $\tilde{\ell}$ & $10.61 \frac{\mathrm{nMol}}{\mu \mathrm{g} \text { lipid protein }}$ & $6.14 \cdot 10^{3}$ & \begin{tabular}{|l|l|l|}
4 & 5 \\
\end{tabular} \\
\hline$f_{\mathrm{Min}}$ & Minimum $f$ for esterification & $36.43 \frac{\mathrm{nMol}}{\mathrm{mg} \text { cell protein }}$ & $1.22 \cdot 10^{10}$ & 4,5 \\
\hline$f_{\mathrm{Max}}$ & Maximum $f$ for esterification & $213.70 \frac{\mathrm{nMol}}{\mathrm{mg} \text { cell protein }}$ & $7.15 \cdot 10^{10}$ & 4,5 \\
\hline$k_{f}$ & Rate of esterification of $f$ & $2.56 \cdot 10^{-2} \frac{1}{\mathrm{~h}}$ & $2.56 \cdot 10^{-2} \frac{1}{\mathrm{~h}}$ & 4,5 \\
\hline$k_{b}$ & Rate of hydrolysis of $b$ & $4.33 \cdot 10^{-2} \frac{\mathrm{I}}{\mathrm{h}}$ & $4.33 \cdot 10^{-2} \frac{1}{h}$ & 45 \\
\hline$c_{f}$ & Rate of efflux of $f$ & $3.32 \cdot 10^{-3} \frac{1}{h}$ & $3.32 \cdot 10^{-3} \frac{1}{h}$ & \begin{tabular}{|l|l|l|}
4 & 5 \\
\end{tabular} \\
\hline$\mu_{f}$ & Rate of efflux of $f$ to $h$ & $1.65 \cdot 10^{-1} \frac{1}{h}$ & $1.65 \cdot 10^{-1} \frac{1}{h}$ & 4,5 \\
\hline$\xi_{f}$ & Saturation of efflux of $f$ to $h$ & $85.41 \frac{\mu \mathrm{g} \text { lipid protein }}{\mathrm{ml}}$ & $3.60 \cdot 10^{11} \frac{1}{\mathrm{~mm}^{3}}$ & 4,5 \\
\hline$n_{f}$ & Exponent of efflux of $f$ to $h$ & $7.37 \cdot 10^{-1}$ & $7.37 \cdot 10^{-1}$ & 45 \\
\hline$f_{\text {In }}$ & $f$ of recruited $m$ & $56.85 \frac{\mathrm{nMol}}{\mathrm{mg} \text { cell protein }}$ & $1.90 \cdot 10^{10}$ & 4, 77 \\
\hline$\mu_{m}$ & Rate of apoptosis of $m$ by $f$ & $9.90 \cdot 10^{-2} \frac{1}{h}$ & $9.90 \cdot 10^{-2} \frac{1}{h}$ & 77 \\
\hline$\xi_{m}$ & Saturation of apoptosis of $m$ by $f$ & $622.8 \frac{\mu \mathrm{g}}{\mathrm{mg} \text { cell protein }}$ & $5.38 \cdot 10^{111}$ & 77 \\
\hline$n_{m}$ & Exponent of apoptosis of $m$ by $f$ & 2.00 & 2.00 & 77 \\
\hline$q_{h, m}$ & Rate of modification of $h$ by $m$ & - & $8.63 \cdot 10^{-6} \frac{\mathrm{mm}^{3}}{\mathrm{~h}}$ & $=\frac{q_{\ell, m}}{7.6}, 11$ \\
\hline$q_{h, e}$ & Rate of modification of $h$ by $e$ & - & $3.26 \cdot 10^{-3} \frac{\mathrm{mm}}{\mathrm{h}}$ & $=\frac{d_{\dot{\ell}, e}}{7.6},[11]$ \\
\hline$H$ & Thickness of early plaque & - & $1.00 \cdot 10^{-2} \mathrm{~mm}$ & $\begin{array}{ll}55 & 76 \\
\end{array}$ \\
\hline$f_{0}$ & Initial $f$ per $m$ & - & $2.85 \cdot 10^{10}$ & $=1.5 f_{\mathrm{In}}$ \\
\hline
\end{tabular}

All units are converted from respective experimental units to the SI units $\mathrm{mm}$ and $\mathrm{h}$ using $\rho_{5}, \rho_{10}$ and $\rho_{14}$ from Table 1 Abbreviations: native low-density lipoproteins, $\ell$; modified low-density lipoproteins, $\tilde{\ell}$; native high-density lipoproteins, $h$; modified high-density lipoproteins, $\tilde{h}$; endothelial cells, $e$; macrophages, $m$; copper sulfate, $\varsigma$; intracellular free cholesterol, $f$; intracellular cholesterol ester, $b$.

covered by a simple analysis utilizing a equidistant grid of the parameter space. The results for varying $r_{h}$ and $r_{m}$ and two different $r_{\ell}$ are visualized in Fig 4 . 

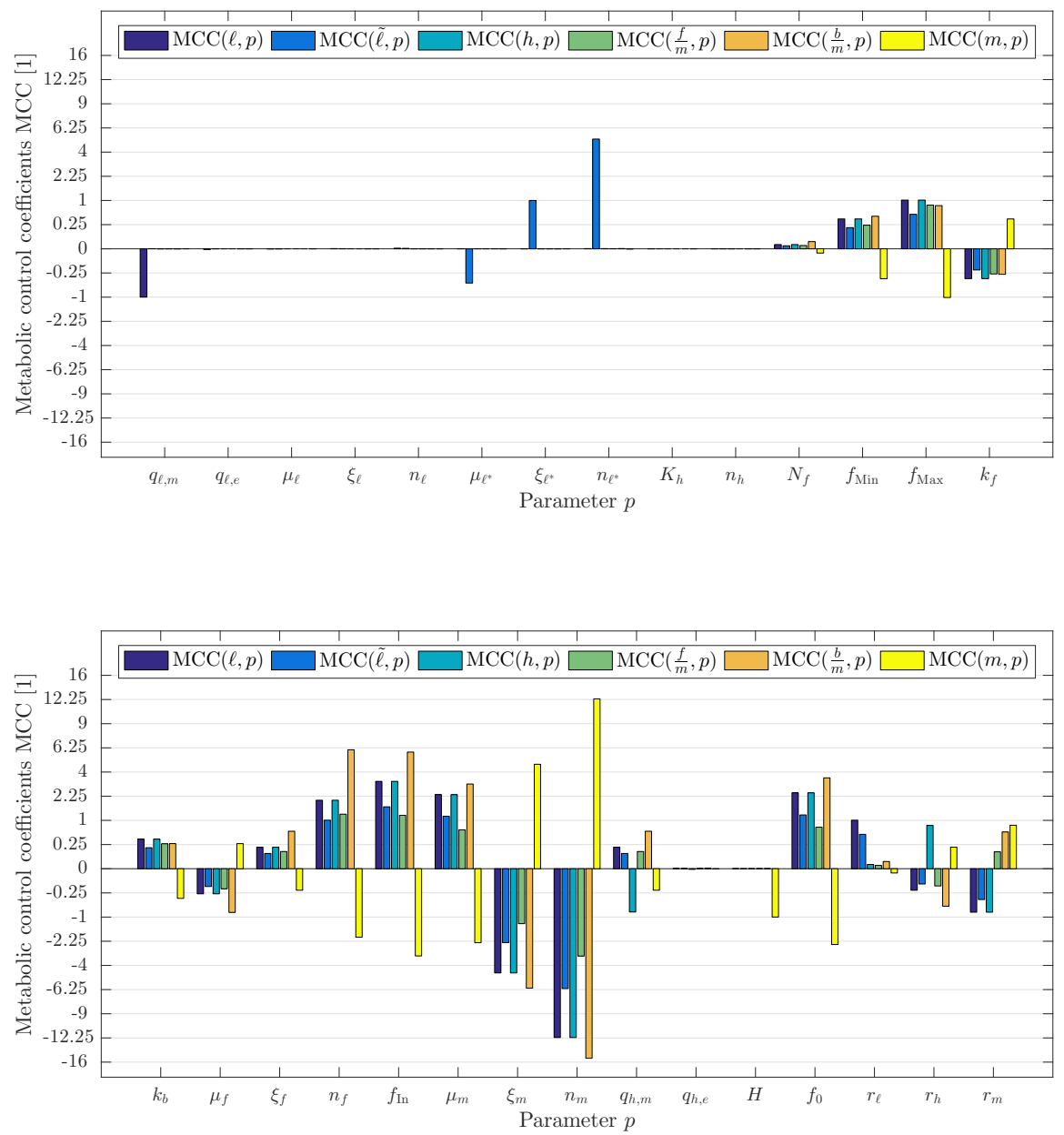

Fig. 2 Metabolic control analysis of the complete mathematical model. The metabolic control coefficients for LDL $\ell$, modLDL $\tilde{\ell}$, HDL $h$, average intracellular free cholesterol per macrophage $\frac{f}{m}$, average intracellular cholesterol ester per macrophage $\frac{f}{m}$ and macrophages $m$ with respect to all parameters $p$ of the complete model are computed as described in the Models and Methods section. For an overview of all parameters of the complete model, see Tables 2 and 4. 
(a)

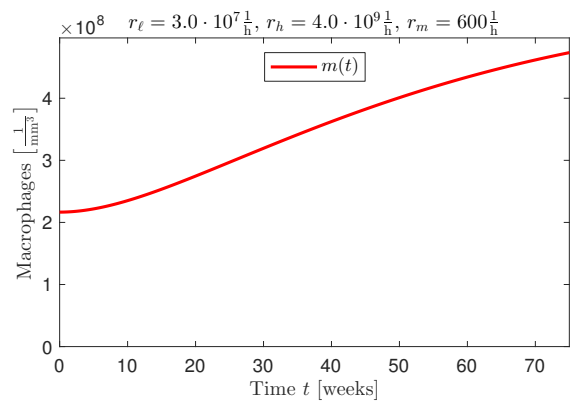

(c)

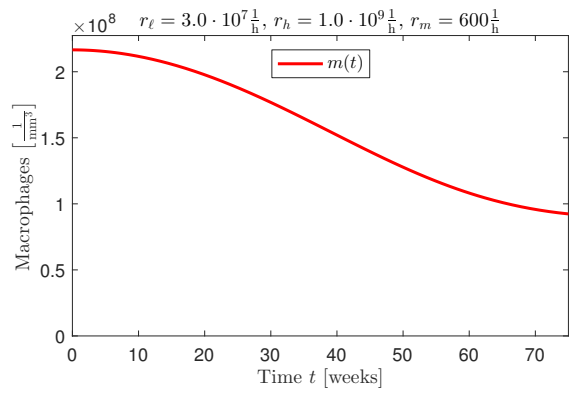

(e)

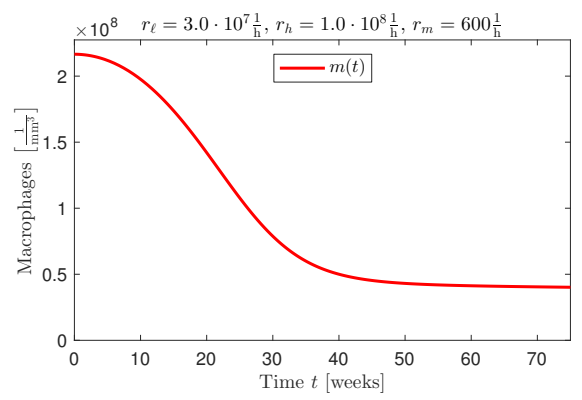

(b)

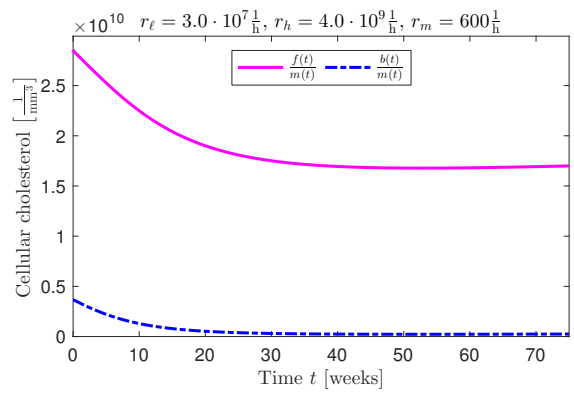

(d)

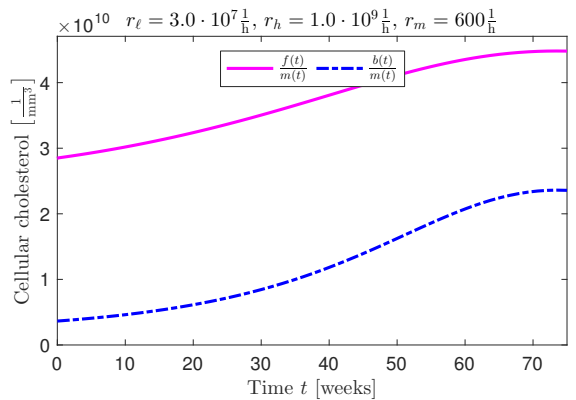

(f)

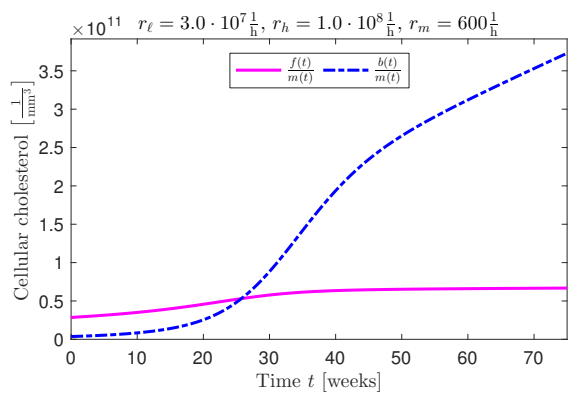

Fig. 3 Examples of densities of macrophages $m(t) \sqrt{(\mathrm{a})(\mathrm{c})(\mathrm{e})}$ and concentrations of intracellular free cholesterol $\frac{f(t)}{m(t)}$ and intracellular choles-

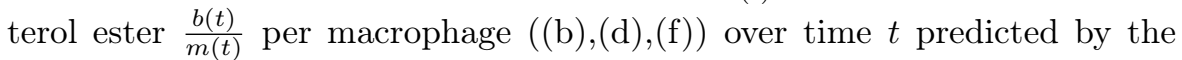
complete mathematical model. Parameters from Table 4 with LDL influx rate $r_{\ell}=3.0 \cdot 10^{7} \frac{1}{\mathrm{~h}}$, macrophage recruitment rate $r_{m}=600 \frac{1}{\mathrm{~h}}$ and varying HDL influx rates $r_{h}=4.0 \cdot 10^{9} \frac{1}{\mathrm{~h}}(\mathrm{a})(\mathrm{b}), \quad r_{h}=1.0 \cdot 10^{9} \frac{1}{\mathrm{~h}}(\mathrm{c})(\mathrm{d})$ and $r_{h}=1.0 \cdot 10^{8} \frac{1}{\mathrm{~h}}(\mathrm{e})(\mathrm{f})$ are used. 
(a)

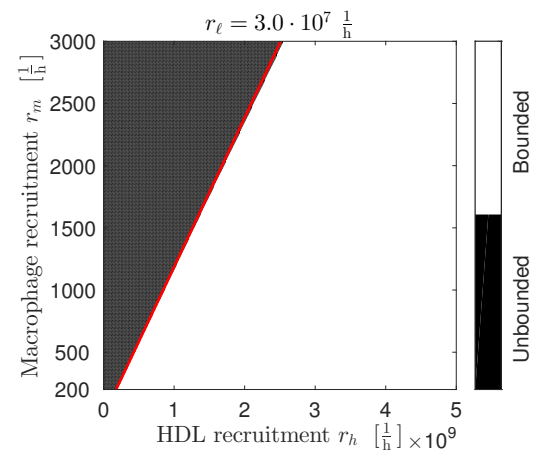

(c)

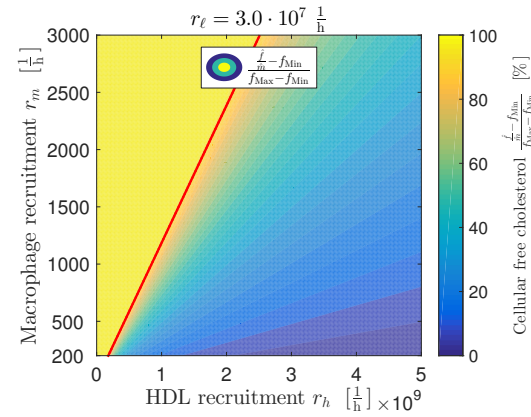

(e)

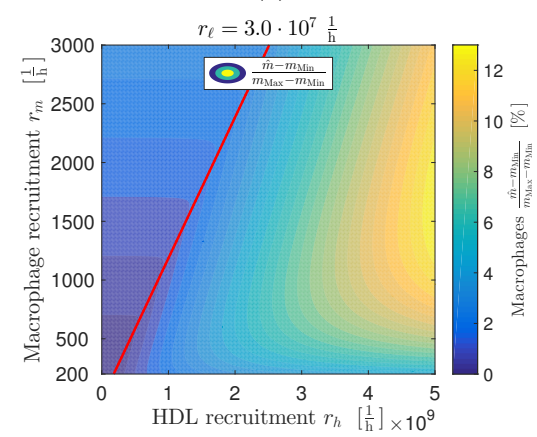

(b)

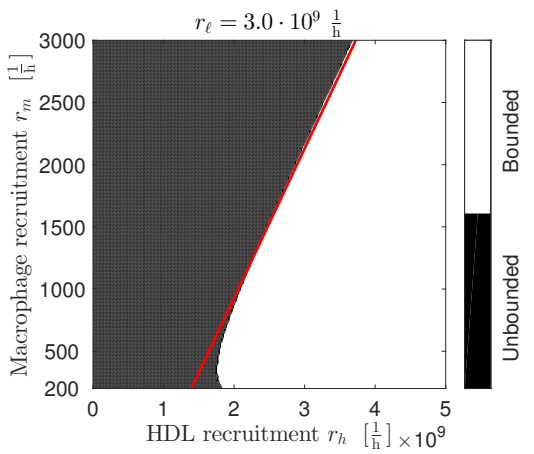

(d)

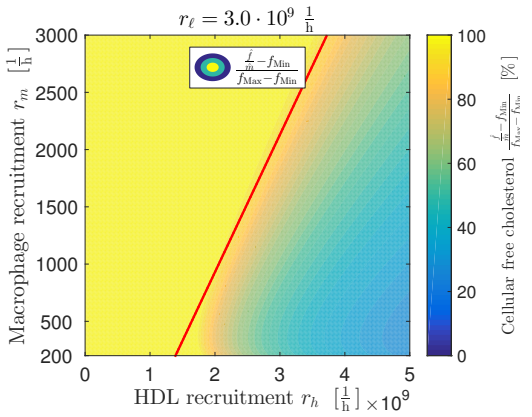

(f)

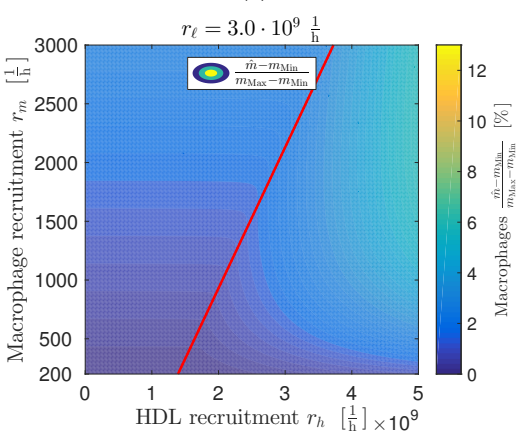

Fig. 4 Stability analysis of the complete mathematical model as described in the Models and Methods section. The boundedness of intracellular cholesterol ester $[(\mathrm{a})(\mathrm{b})]$, the steady-state concentration of intracellular free cholesterol per macrophages $\frac{\hat{f}}{\hat{m}}$ relative to $\left[f_{\mathrm{Min}} ; f_{\mathrm{Max}}\right](\mathrm{c})(\mathrm{d})$ and the steady-state density of macrophages $\hat{m}$ relative to $\left[m_{\mathrm{Min}} ; m_{\mathrm{Max}}\right]($ (e) (f) $)$ are predicted for varying recruitment rates of HDL $r_{h}$ and macrophages $r_{m}$. The recruitment rates of LDL are $r_{\ell}=r_{\ell}=3.0 \cdot 10^{7} \frac{1}{\mathrm{~h}} \sqrt{(\mathrm{a})(\mathrm{c})(\mathrm{e})}$ and $r_{\ell}=3.0 \cdot 10^{9} \frac{1}{\mathrm{~h}} \sqrt{(\mathrm{b})(\mathrm{d})(\mathrm{f})}$, respectively. The red line indicates the approximate boundary between where intracellular cholesterol ester concentrations change from being bounded to unbounded. Its equation is $1.2 \cdot 10^{-6} r_{h}=r_{m}+4.9 \cdot 10^{-7} r_{\ell}$. 


\section{Discussion}

Three mathematical submodels of in vitro systems are developed and their parameters least-squares fitted to measurements of various experimental setups, see Table 3. The fitted submodels show very good agreement with the experimental data, see Appendix 2: Least-squares fits of submodels. The fitted parameters given in Table 4 lead to the following observations.

- The ingestion of native and modified LDL follow a very similar saturating kinematic. The rate of ingestion of native LDL $\mu_{\ell}$ is approximately 5 times smaller than the rate of ingestion of modified LDL $\mu_{\tilde{\ell}}$. This is consistent with literature 63.40.

- Using a density of $1205.2 \frac{1}{\mathrm{~mm}^{2}}$ [29] of endothelial cells per unit endothelial monolayer area the rate of oxidative modification of native LDL by endothelial cells can be compared to the modification rate by macrophages. The rate of modification of native LDL by macrophages $q_{\ell, m}$ is approximately 3 times smaller than the modification rate by endothelial cells $q_{\ell, e}$.

- Free cholesterol and cholesterol ester are in a buffer-like relationship, where the maximum concentration of intracellular cholesterol $f_{\mathrm{Max}}$ is approximately 6 times bigger than the minimum concentration $f_{\text {Min }}$. The rate of esterification of intracellular cholesterol $k_{f}$ varies as the level of intracellular free cholesterol changes. However, the average rate of esterification of cholesterol $k_{f}$ is approximately 2 times faster than the rate of hydrolysis $k_{b}$. In the absence of a source and acceptor for the intracellular free cholesterol the model suggests that approximately $63.8 \%$ of the stored cholesterol is hydrolyzed and re-esterified each day which is moderately higher than $50 \%$ as proposed in 5 .

- The dynamic of lipids and cholesterols, i.e. of LDL, modLDL, HDL, free cholesterol and cholesterol ester adapts very rapidly to the current number of macrophages and is in the order of minutes to hours. In contrast, the fate of macrophages is determined on a much larger time scale in the order of weeks to months. Hence, the long-term outcome for the plaque is driven by the fate of macrophages, which is governed by the short term quasiequilibrium concentrations of intracellular cholesterols. The concentrations of intracellular cholesterols, however, are governed by the uptake of modLDL and in particular the efflux to available native HDL which are determined by the very rapid influx through the endothelium and the modification by macrophages.

We have derived a mathematical model for the early development of atherosclerotic plaques. It is based on submodels of in vitro systems and most importantly is parameterized using data from in vitro experimental studies. The complete model includes and quantifies the following biological processes: oxidative modification of native LDL and HDL by endothelial cells and macrophages, protection against LDL modification by HDL, ingestion of native and modified LDL by macrophages, esterification and hydrolysis of intracellular cholesterols, 
cholesterol efflux from macrophages to HDL, apoptosis of macrophages as well as the recruitment of LDL, HDL and macrophages into the plaque.

The sensitivity analysis of the complete model with respect to its parameters that we performed reveals that the complete model is especially sensitive to parameters connected to the apoptosis of macrophages (e.g. $\left.\mu_{m}, \xi_{m}, n_{m}\right)$ and to free cholesterol (e.g. $f_{\mathrm{Max}}, f_{\mathrm{In}}, f_{0}$ ), see Fig 2 . The sensitivity of the complete model with respect to free cholesterol-related parameters is high but their estimation is based on a large number of data points, see Table 3 and so their value is likely to be reliable. However, the high sensitivity with respect to the parameters of macrophage apoptosis $n_{m}$ and $\xi_{m}$ is crucial. This sensitivity is approximately 3 times bigger compared to the sensitivity of other parameters and additionally the parameters of macrophage apoptosis are estimated only on the base of two data points, see Table 3 More suitable experimental data from literature could not be found and hence further experimental work quantifying apoptosis of macrophages in atherosclerotic plaques is recommended. The sensitivity analysis and further investigations reveal that the influence of the rates of ingestion of native LDL as well as HDL protection against native LDL modification are minor to the plaque development process and plaque outcome in the model. In both cases this is due to the low concentrations of native LDL and HDL compared to the respective saturation coefficients.

The complete model is able to predict three qualitatively different plaque developments. They are characterized by the following long-term behaviours:

- Regressing plaque or non-atherosclerotic region: all concentrations are bounded and the long-term equilibrium concentrations are characterized by low modLDL $\hat{\tilde{\ell}}$, high macrophages $\hat{m}$, low intracellular free cholesterol per macrophage $\frac{\hat{f}}{\hat{m}}$ and very low intracellular cholesterol ester per macrophage $\frac{\hat{b}}{\hat{m}}$. Macrophages exhibit a low rate of apoptosis and the ingested modLDL within macrophages is effectively offloaded to native HDL. An example of time-dependent concentrations of macrophage and intracellular cholesterols for a regressing plaque is shown in Fig [a (a) and a (b).

- Stable atherosclerotic plaque: all concentrations are bounded and the longterm equilibrium concentrations are characterized by moderate modLDL $\hat{\tilde{\ell}}$, low macrophages $\hat{m}$, moderate intracellular free cholesterol per macrophage $\frac{\hat{f}}{\hat{m}}$ and low intracellular cholesterol ester per macrophage $\frac{\hat{b}}{\hat{m}}$. Macrophages exhibit a moderate rate of apoptosis and the ingested modLDL within macrophages is still offloaded to native HDL. An example of time-dependent concentrations of macrophage and intracellular cholesterols for a stable plaque is shown in Fig $3(\mathrm{~s})$ and $3(\mathrm{~s})$.

- Unstable atherosclerotic plaque: concentration of intracellular cholesterol ester per macrophage $\frac{b}{m}$ is unbounded and the remaining long-term equilibrium concentrations are characterized by high modLDL $\hat{\tilde{\ell}}$, very low macrophages $\hat{m}$ and very high intracellular free cholesterol per macrophage $\frac{\hat{f}}{\hat{m}}$. Macrophages exhibit a high rate of apoptosis and not all of the ingested modLDL within macrophages is offloaded to native HDL. An example of 
time-dependent concentrations of macrophage and intracellular cholesterols

for a unstable plaque is shown in Fig [3(e) and $3(\mathrm{f})$

The distinction between mathematically stable and unstable model plaques is clear, whereas there is a smooth transition between regressing and stable plaques. The definitions of mathematical stability of model plaques closely align with medical definitions of plaques that become clinically stable or unstable 35, 61, 62.

The recruitment rates of LDL $r_{\ell}$, HDL $r_{h}$ and macrophages $r_{m}$ which characterize the plaque under consideration show only little influence in the performed local sensitivity analysis, see Fig 2. Still, the long-term behavior of the complete model and hence the classification of plaques into the three categories above is determined by them as they can vary by an order of up to 1000 , see Table 2. The stability analysis visualized in Fig 4 shows that a model plaque is unstable if the recruitment of HDL $r_{h}$ is low and the recruitment of macrophages $r_{m}$ is high. More precisely a plaque is unstable if

$$
1.2 \cdot 10^{-6} r_{h}<r_{m}+4.9 \cdot 10^{-7} r_{\ell}
$$

holds approximately, see Fig $3(\mathrm{a})$ and Fig $3(\mathrm{~b})$ A plaque is regressing if the recruitment rate of macrophages $r_{m}$ is low and the recruitment rate of HDL $r_{h}$ is sufficiently high, see Fig $3(\mathrm{c}), 3(\mathrm{~d}), 3(\mathrm{e})$ and $3(\mathrm{f})$. For increasing $r_{m}$ or decreasing $r_{h}$ the plaque smoothly transitions from a regressing to a stable atherosclerotic plaque. In general, an increased recruitment rate of LDL $r_{\ell}$ results in a less stable atherosclerotic plaque, but the predicted influence of $r_{\ell}$ is small compared to $r_{h}$ and $r_{m}$. However, the rate of macrophage recruitment $r_{m}$ in reality is a function of modLDL $\tilde{\ell}$ (which, in turn, is strongly dependent on $r_{\ell}$ ), since it triggers the production of vascular cell adhesion molecules and monocyte chemoattractant proteins by endothelial cells which determine the number of macrophages recruited from the blood [22,29, 1]. Thus, the rate of macrophage recruitment is actually dependent on the LDL recruitment. Therefore, the rate of macrophage recruitment that is used in this model to determine plaque stability is, in effect, the rate of macrophage recruitment at the long-term steady state equilibrium, i.e. $r_{m}=r_{m}\left(\hat{\tilde{\ell}}\left(r_{\ell}\right)\right)$. As this dependency is not explicitly considered but $r_{m}$ is a fixed input parameter of the complete model, the influence of the rate of LDL recruitment $r_{\ell}$ is underestimated. The explicit consideration of this dependency as well as the explicit modeling of the driving mechanisms for the recruitment rates of LDL $r_{\ell}$, HDL $r_{h}$ and macrophages $r_{m}$ will be addressed in future work. Also we have not included the increase of the thickness of the plaque $H$ due to the advancing accumulation of macrophages, nor, indeed any other spatially-dependent effect. Hence, the reduction of the recruitment rates of LDL, HDL and macrophage by the factor $\frac{1}{H}$ which will continually decrease in growing plaques is neglected. This leads to an overestimation of all three rates and will be addressed in future work too.

The mathematical stability criterion given by equation 10 is not suitable for the experimental determination of the clinical stability of a given atherosclerotic plaque in vivo. Macrophages within unstable plaques contain a very high 
concentration of intracellular cholesterol ester, see 3(a), 3(b), 3(c) and 3(d), As a consequence, the predicted concentration of intracellular free cholesterol is also high in unstable plaques due to the buffer-like behaviour between free and esterified cholesterol. More precisely the estimated concentration of intracellular free cholesterol in model plaques is close to $f_{\mathrm{Max}} \approx 213.70 \frac{\mathrm{nMol}}{\mathrm{mg} \text { cell protein }}$ when the plaque becomes unstable in the long-term. This suggests that a high concentration of intracellular free cholesterol within plaque macrophages is necessary in order for a plaque to become clinically unstable in the future. The relation between intracellular free cholesterol and plaque growth has yet not been investigated experimentally to our knowledge.

This model assumes that macrophages only undergo recruitment and apoptosis and do not experience necrosis, proliferation and egress from the plaque. This assumption is valid only for early stages of the atherosclerotic process, i.e. for types $I$ and $I I$ atherosclerosis, see 61. In contrast to early stages, necrosis, proliferation and egress are important in advanced lesions $66,57,33$, 54 and lead to a reduction of cholesterol ester within macrophages in latestage plaques. If the processes of late-stage plaques are included in the model, in addition to recruitment and apoptosis of macrophages, the behaviour of atherosclerotic plaques of types $I I I$ and $I V$ can be modelled. As for apoptosis, there is very little experimental data suited for parameter estimations for rates of necrosis, proliferation and egress of macrophages. The extension of the model presented here to represent the behaviour and fate of macrophages in late stage plaques, requires further work. In particular, experiments are needed to quantify macrophage behaviour in late-stage plaques.

\section{Conclusion}

Three mathematical submodels of in vitro systems were developed and their parameters estimated from experimental studies. These submodels closely reproduced the experimental results. The three submodels that were closely connected to in vitro studies were combined to a quantified and predictive complete mathematical model of early atherosclerotic plaques which uses the physiological parameters determined by the submodels. The analysis of the complete model suggests that further experimental work quantifying the different fates of macrophages as a function of cholesterol load and the balance between free cholesterol and cholesterol ester may give valuable insight into long-term plaque outcomes. We used the complete model to predict the longterm stability of early atherosclerotic plaques and to classify their stability with respect to their rates of recruitment of LDL, HDL and macrophages. The extension to predict the behaviour of later stages of atherosclerosis is not trivial and requires further experimental and modeling work.

Acknowledgements We thank Dr. Christina Bursill of the South Australian Health and Medical Research Institute for helpful discussions. This work was supported by the German Research Foundation (DFG) and the Technical University of Munich (TUM) in the 
framework of the Open Access Publishing Program. Michael W. Gee and Moritz P. Thon acknowledge the financial support given by the International Graduate School of Science and Engineering of the TUM under the project BioMat01, A Multiscale Model of Atherosclerosis. Mary R. Myerscough and Hugh Z. Ford acknowledge support from an Australian Research Council Discovery Project Grant (to Mary R. Myerscough).

Conflict of Interest All authors declare that no conflicts of interest exist.

\section{Appendix 1: Experiment-specific parameters of submodels}

The following Tables 5, 6 and 7 contain the experiment-specific parameters of the mathematical submodels of in vitro systems in analogy to the experimental setups in [27, $32,41,4,5]$.

Table 5 Experiment-specific parameters of the mathematical submodel of LDL modification and ingestion (submodel 1) in analogy to the experimental setups in [27] and 32 .

\begin{tabular}{|c|c|c|c|c|c|c|c|c|}
\hline Experiment & $\frac{\Xi}{\Xi}$ & 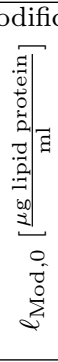 & 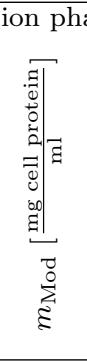 & 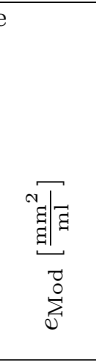 & 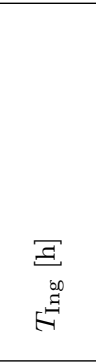 & 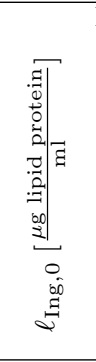 & gestion phase & 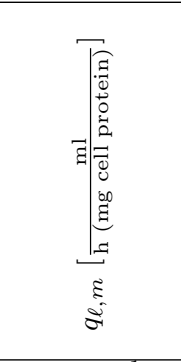 \\
\hline 27], Fig $1, e_{\mathrm{Mod}}=0$ & 24 & 200 & 0 & 0 & $0-20$ & 10 & $4.16 \cdot 10^{-2 *}$ & $1.18 \cdot 10^{-1 * *}$ \\
\hline 27, Fig $1, e_{\mathrm{Mod}}=1413.7$ & 24 & 200 & 0 & 1413.7 & $0-20$ & 10 & $4.16 \cdot 10^{-2 *}$ & $1.18 \cdot 10^{-1 * *}$ \\
\hline 27 , Fig $2, e_{\mathrm{Mod}}=0$ & $0-46$ & 200 & 0 & 0 & 5 & 8.5 & $4.16 \cdot 10^{-2 *}$ & $1.18 \cdot 10^{-1 * *}$ \\
\hline 27, Fig $2, e_{\mathrm{Mod}}=1413.7$ & $0-46$ & 200 & 0 & 1413.7 & 5 & 8.5 & $4.16 \cdot 10^{-2 *}$ & $1.18 \cdot 10^{-1 * *}$ \\
\hline 27, Fig $5, e_{\mathrm{Mod}}=0$ & 26 & 200 & 0 & 0 & 5 & $0-40$ & $4.16 \cdot 10^{-2 *}$ & $1.18 \cdot 10^{-1 * *}$ \\
\hline 27, Fig $5, e_{\mathrm{Mod}}=1413.7$ & 26 & 200 & 0 & 1413.7 & 5 & $0-40$ & $4.16 \cdot 10^{-2 *}$ & $1.18 \cdot 10^{-1 * *}$ \\
\hline 32, Fig $1 \mathrm{a}, m_{\mathrm{Mod}}=0$ & $0-24$ & 100 & 0 & 0 & 24 & 10 & $5.54 \cdot 10^{-1 *}$ & 0 \\
\hline 32 , Fig $1 \mathrm{a}, m_{\mathrm{Mod}}=1.11$ & $0-24$ & 100 & $1.11 *$ & 0 & 24 & 10 & $5.54 \cdot 10^{-1 *}$ & 0 \\
\hline 32 , Fig $4, m_{\mathrm{Mod}}=0$ & 24 & 100 & 0 & 0 & 24 & $0-50$ & $5.54 \cdot 10^{-1 *}$ & 0 \\
\hline 32, Fig $4, m_{\mathrm{Mod}}=1.11$ & 24 & 100 & $1.11 *$ & 0 & 24 & $0-50$ & $5.54 \cdot 10^{-1 *}$ & 0 \\
\hline
\end{tabular}

Parameters indicated by ${ }^{*}$ are estimated using $\rho_{5}$ from Table 1 . Parameters indicated by ** are estimated by least-squares fitting to the experimental results. All values are given in the units of submodel 1. 
Table 6 Experiment-specific parameters of the mathematical submodel of HDL protection against LDL modification (submodel 2) in analogy to the experimental setups in 41 .

\begin{tabular}{|l||c|c|c|c|}
\hline Quantity & $T_{\text {Mod }}[\mathrm{h}]$ & $\ell_{0}\left[\frac{\mathrm{mg} \text { protein }}{\mathrm{ml}}\right]$ & $h_{0}\left[\frac{\mathrm{mg} \text { protein }}{\mathrm{ml}}\right]$ & $\varsigma\left[\frac{\mu \text { Mol }}{\mathrm{ml}}\right]$ \\
\hline Experiment, Fig 4, $\ell_{0}=0, h_{0}=1.5$ & $0-24$ & 0 & 1.5 & 5 \\
41], Fig 4, $\ell_{0}=1.5, h_{0}=0$ & $0-24$ & 1.5 & 0 & 5 \\
41], Fig 4, $\ell_{0}=1.5, h_{0}=1.5$ & $0-24$ & 1.5 & 1.5 & 5 \\
41], Fig 5 & 6 & 1.5 & $0-2$ & 5 \\
\hline
\end{tabular}

All values are given in the units of submodel 2 .

Table 7 Experiment-specific parameters of the mathematical submodel of cholesterol cycle and reverse cholesterol transport (submodel 3) in analogy to the experimental setups in [4] and [5].

\begin{tabular}{|c|c|c|c|c|c|c|c|}
\hline 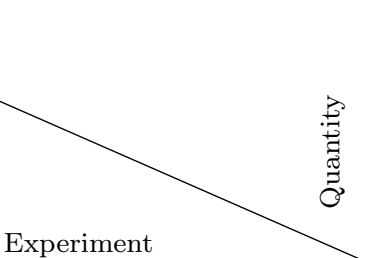 & 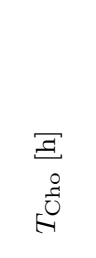 & $\begin{array}{l}\Xi \\
\vec{E}\end{array}$ & 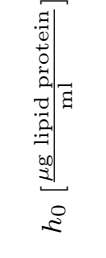 & 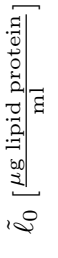 & 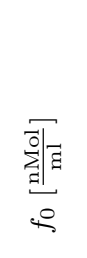 & 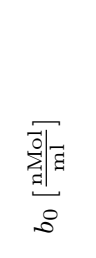 & 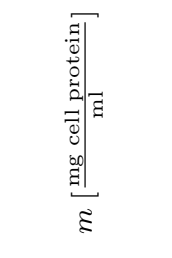 \\
\hline [4, Fig 1a \& 1b, $\tilde{\ell}_{0}=0$ & $0-96$ & 0 & 0 & 0 & 56 & 0 & $4.16 \cdot 10^{-1 *}$ \\
\hline [4, Fig $1 \mathrm{a} \& 1 \mathrm{~b}, \tilde{\ell}_{0}=25$ & $0-96$ & 0 & 0 & 25 & 56 & 0 & $4.16 \cdot 10^{-1 *}$ \\
\hline [5], Fig $1 \mathrm{a}, 1 \mathrm{~b} \& 1 \mathrm{c}, h_{0}=0$ & $0-72$ & 0 & 0 & 0 & 183 & 342 & $6.93 \cdot 10^{-1 *}$ \\
\hline [5], Fig 1a, 1b \& 1c, $T_{h}=0$ & $0-24$ & 0 & 250 & 0 & 183 & 342 & $6.93 \cdot 10^{-1 *}$ \\
\hline [5], Fig 1a, 1b \& 1c, $T_{h}=24$ & $0-48$ & 24 & 250 & 0 & 183 & 342 & $6.93 \cdot 10^{-1 *}$ \\
\hline [5], Fig 1a, 1b \& 1c, $T_{h}=48$ & $0-72$ & 48 & 250 & 0 & 183 & 342 & $6.93 \cdot 10^{-1 *}$ \\
\hline [5], Fig $2 \mathrm{a}, T_{\mathrm{Cho}}=8$ & 8 & 0 & $0-267$ & 0 & 212 & 405 & $6.93 \cdot 10^{-1 *}$ \\
\hline [5], Fig $2 \mathrm{a}, T_{\mathrm{Cho}}=24$ & 24 & 0 & $0-267$ & 0 & 212 & 405 & $6.93 \cdot 10^{-1 *}$ \\
\hline$[5$, Fig 4 & 24 & 0 & $0-250$ & 0 & $177^{* *}$ & $456^{* *}$ & $1.39 *$ \\
\hline 5, Fig $7 \mathrm{a} \& 7 \mathrm{~b}, h_{0}=0$ & $0-6$ & 0 & 0 & 0 & 190 & 488 & $6.93 \cdot 10^{-1 *}$ \\
\hline$[5]$, Fig $7 \mathrm{a} \& 7 \mathrm{~b}, h_{0}=200$ & $0-6$ & 0 & 200 & 0 & 190 & 488 & $6.93 \cdot 10^{-1 *}$ \\
\hline
\end{tabular}

Parameters indicated by ${ }^{*}$ are estimated using $\rho_{5}$ from Table 1 . Parameters indicated by ** are estimated by least-squares fitting to the experimental results. All values are given in the units of submodel 3 .

\section{Appendix 2: Least-squares fits of submodels}

The following Figures 5, 6, 7, 8, 9 and 10 contain all least-squares fits of the mathematical submodels of in vitro systems to experimental results in [27, 32, 41,4, 5, 77. Appendix 1: Experiment-specific parameters of submodels and Table 4 give the experiment-specific and least-squares fitted parameters of the submodels, respectively. 
(a)

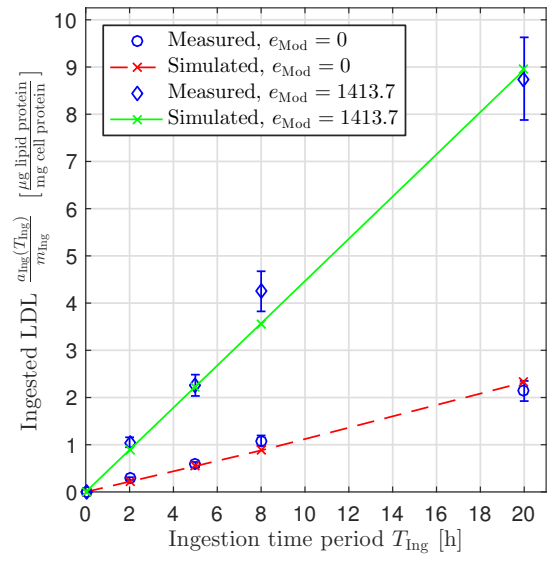

(b)

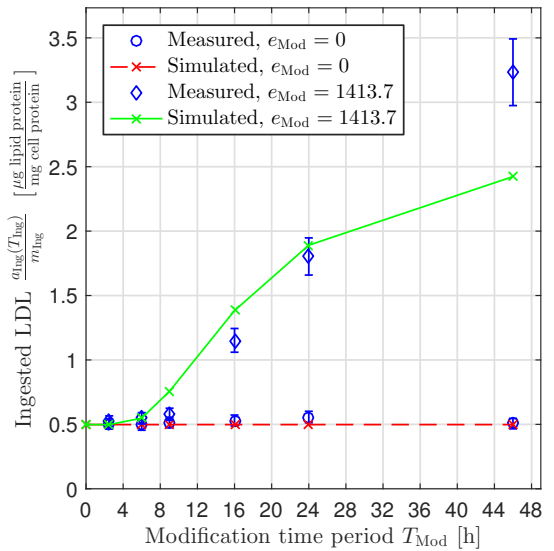

(c)

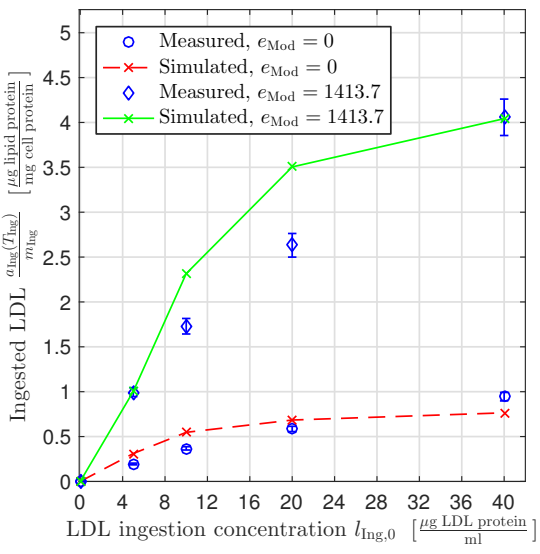

Fig. 5 Comparison of results of the mathematical submodel of LDL modification and ingestion (submodel 1) with results from various experimental setups in [27]. Least-squares fits of the simulated ingestion of native and modified LDL per macrophage $\frac{a_{\text {Ing }}\left(T_{\text {Ing }}\right)}{m_{\text {Ing }}}$ to experimental results (a) in [27, Fig 1 for varying ingestion time periods $T_{\text {Ing }},(\mathrm{b})$ in [27, Fig 2 for varying modification time periods $T_{\text {Mod }}$ and (c)] in [27], Fig 5 for varying initial LDL ingestion concentrations $\ell_{\text {Ing, }, 0}$. 
(a)

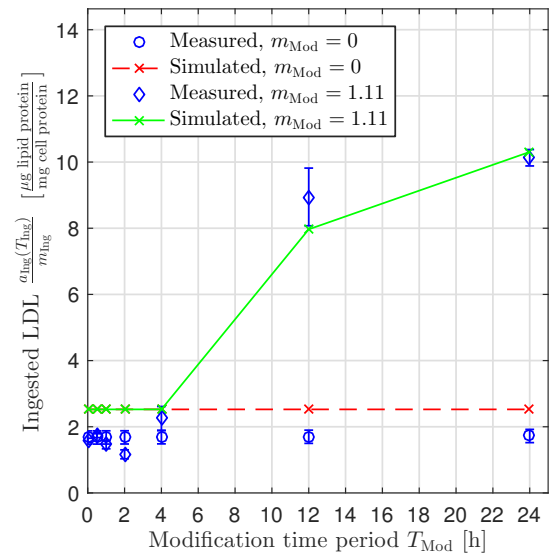

(b)

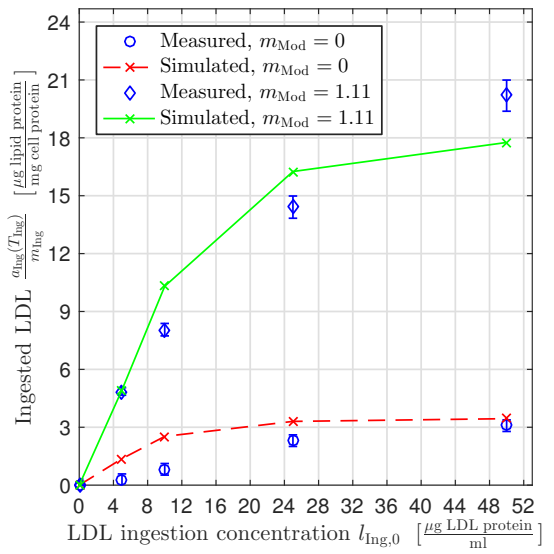

Fig. 6 Comparison of results of the mathematical submodel of LDL modification and ingestion (submodel 1) with results from various experimental setups in 32. Least-squares fits of the simulated ingestion of native and modified LDL per macrophage $\frac{a_{\text {Ing }}\left(T_{\text {Ing }}\right)}{m_{\text {Ing }}}$ to experimental results (a) in [32, Fig $1 a$ for varying modification time periods $T_{\mathrm{Mod}}$ and (b) in [32, Fig 4 for varying initial LDL ingestion concentrations $\ell_{\text {Ing, } 0}$.

(a)

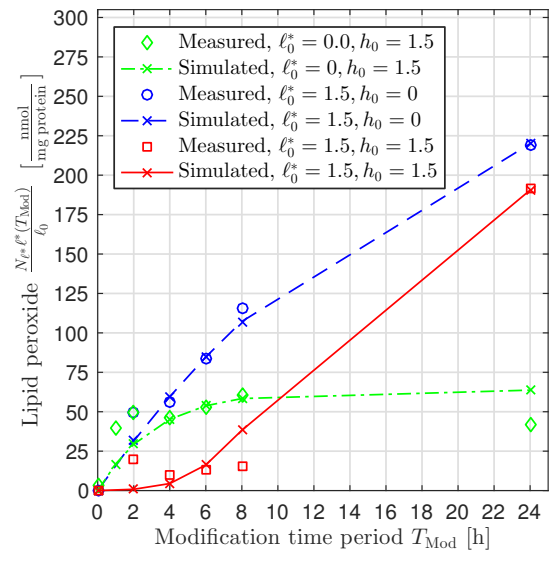

(b)

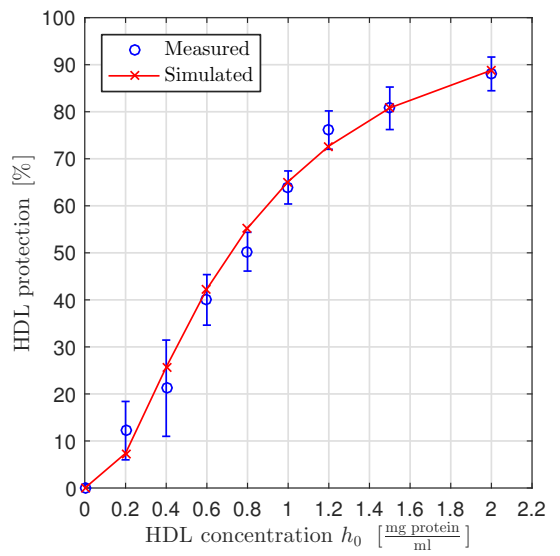

Fig. 7 Comparison of results of the mathematical submodel of the HDL protection against LDL modification (submodel 2) with results from various experimental setups in [41. Least-squares fits of the simulated lipid peroxide content per lipoprotein $\frac{N_{\tilde{\ell}} \tilde{\ell}\left(T_{\mathrm{Mod}}\right)}{\ell_{0}}$ and the HDL protection to experimental results (a) in [41, Fig 4 for varying modification time periods $T_{\mathrm{Mod}}$ and (b) in 41], Fig 5 for varying initial HDL concentrations $h_{0}$. 
(a)

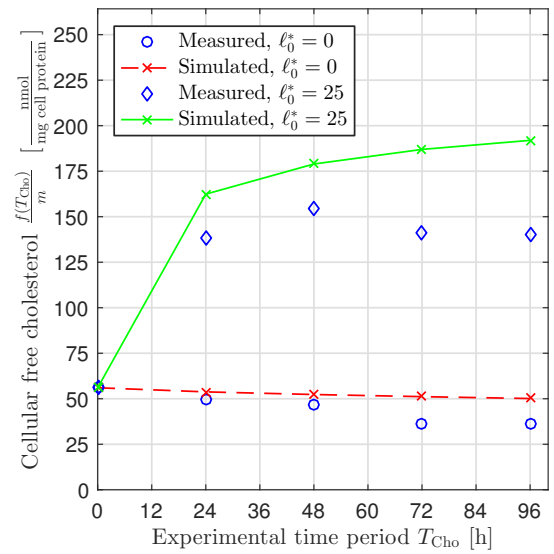

(b)

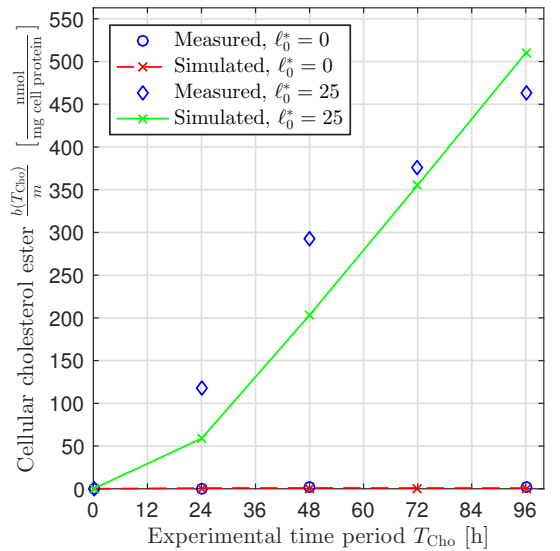

Fig. 8 Comparison of results of the mathematical submodel of cholesterol cycle and reverse cholesterol transport (submodel 3) with results from various experimental setups in [4]. Least-squares fits of the simulated concentration of intracellular free cholesterol $\frac{f\left(T_{\mathrm{Cho}}\right)}{m}$ to the experimental results (a) in 4 , Fig $1 \mathrm{a}$ and (b) in [4, Fig $1 \mathrm{~b}$ for varying experimental time periods $T_{\text {Cho. }}$.

(a)

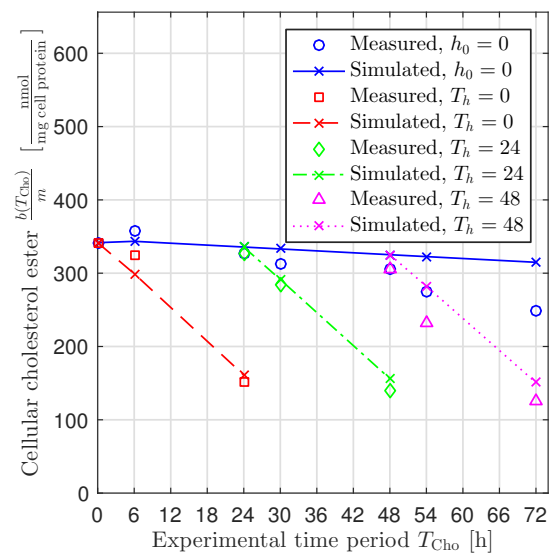

(b)

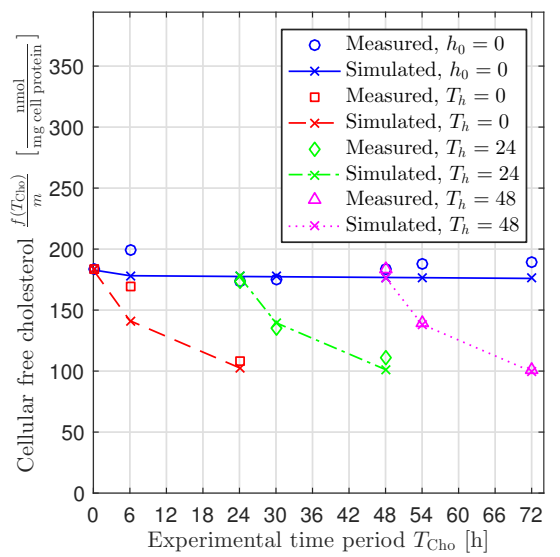

Fig. 9 Comparison of results of the mathematical submodel of cholesterol cycle and reverse cholesterol transport (submodel 3) with results from various experimental setups in [5]. Least-squares fits of the simulated concentration of intracellular free cholesterol $\frac{f\left(T_{\mathrm{Cho}}\right)}{m}$, intracellular cholesterol ester $\frac{b\left(T_{\mathrm{Cho}}\right)}{m}$ and excreted cholesterol $\frac{r\left(T_{\mathrm{Cho}}\right)}{m}$ per macrophage to the experimental results (a) in [5], Fig 1a for varying experimental time periods $T_{\mathrm{Cho}},(\mathrm{b})$ in [5], Fig 1b for varying experimental time periods $T_{\mathrm{Cho}}$. 
(c)

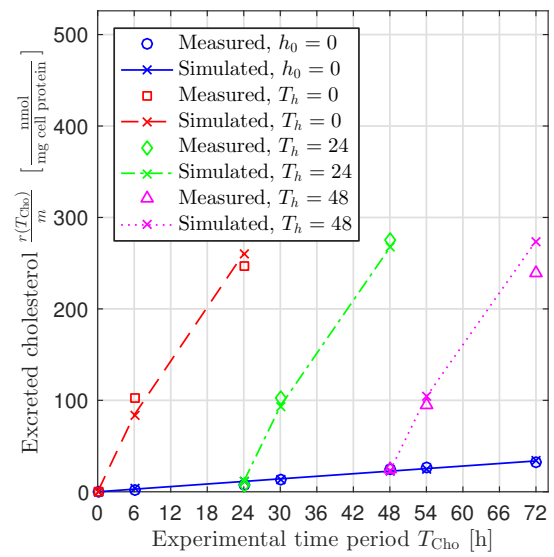

(e)

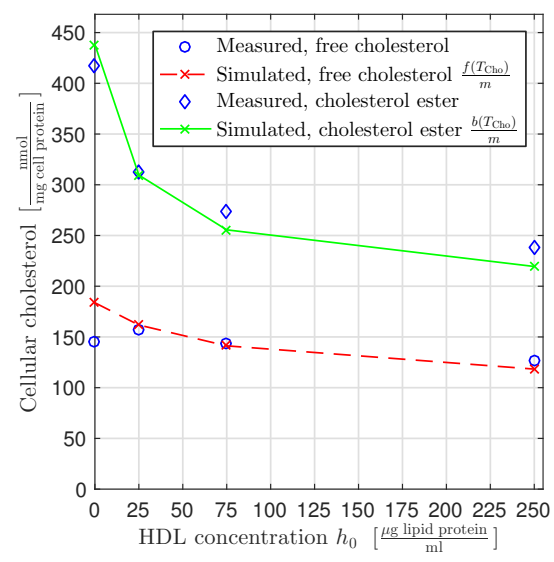

(d)

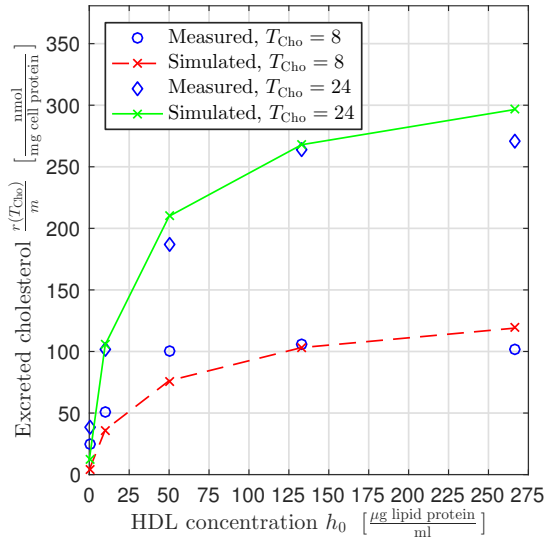

(f)

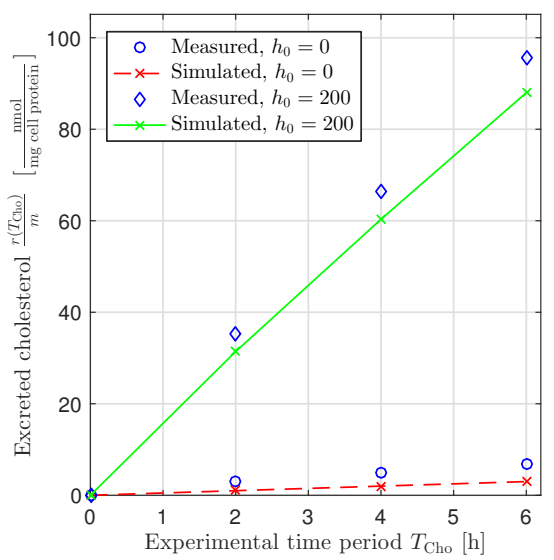

Fig. 9 Comparison of results of the mathematical submodel of cholesterol cycle and reverse cholesterol transport (submodel 3) with results from various experimental setups in [5]. Least-squares fits of the simulated concentration of intracellular free cholesterol $\frac{f\left(T_{\text {Cho }}\right)}{m}$, intracellular cholesterol ester $\frac{b\left(T_{\text {Cho }}\right)}{m}$ and excreted cholesterol $\frac{r\left(T_{\mathrm{Cho}}\right)}{m}$ per macrophage to the experimental results (c) in [5], Fig 1c for varying experimental time periods $T_{\mathrm{Cho}},(\mathrm{d})$ in [5], Fig $2 \mathrm{a}$ for varying additions of HDL $h_{0},(\mathrm{e})$ in [5], Fig 4 for varying additions of HDL $h_{0}$ and (f) in [5], Fig $7 \mathrm{~b}$ for varying experimental time periods $T_{\mathrm{Cho}}$. 


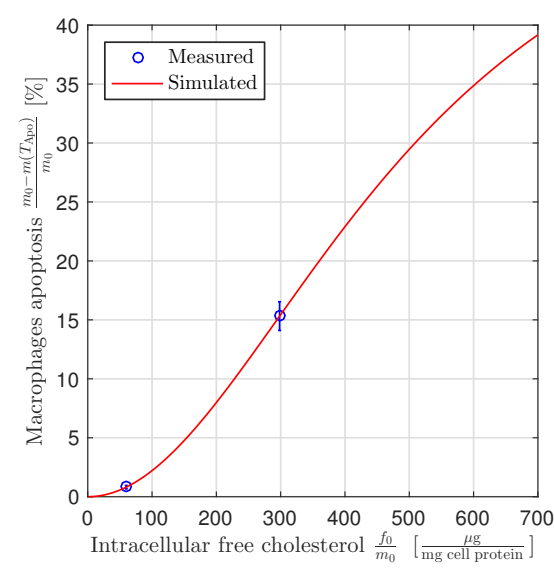

Fig. 10 Comparison of least-squares fit of simulated macrophage apoptosis to experimental results in [77, Fig 4a.

\section{Appendix 3: Proof of Proposition 1}

In the following we prove Proposition 1 . To prove the positivity of $\ell$, i.e. $\ell(t) \geq 0 \forall t \geq 0$ it is sufficient to note that $\ell(0)=0$ and that $\ell(t)=0$ implies

$$
\frac{\mathrm{d}}{\mathrm{d} t} \ell(t) \stackrel{6}{=} \frac{r_{\ell}}{H}>0 \text {. }
$$

due to the strict positivity of the parameters. The positivity of $h, m$ and $\tilde{\ell}$ can be proved in an analogue fashion. Since $\frac{f(0)}{m(0)}=f_{0} \geq f_{\mathrm{Min}}$ and $\frac{f(t)}{m(t)}=f_{\mathrm{Min}}$ implies

$$
\frac{\mathrm{d}}{\mathrm{d} t}\left(\frac{f(t)}{m(t)}\right)=\frac{\frac{\mathrm{d}}{\mathrm{d} t} f(t)-\frac{f(t)}{m(t)} \frac{\mathrm{d}}{\mathrm{d} t} m(t)}{m(t)} \stackrel{\frac{6}{6}}{\geq} \frac{\frac{r_{m}}{H} f_{\mathrm{In}}-f_{\operatorname{Min}} \frac{r_{m}}{H}}{m(t)} \geq 0,
$$

it holds $\frac{f(t)}{m(t)} \geq f_{\text {Min }} \forall t \geq 0$. This also implies the positivity of $f$. Given that $\frac{f(0)}{m(0)}=f_{0}<f_{\text {Max }}$ and that $\frac{f(t)}{m(t)} \rightarrow f_{\text {Max }}$ implies

$$
\frac{\mathrm{d}}{\mathrm{d} t}\left(\frac{f(t)}{m(t)}\right)=\frac{\frac{\mathrm{d}}{\mathrm{d} t} f(t)-\frac{f(t)}{m(t)} \frac{\mathrm{d}}{\mathrm{d} t} m(t)}{m(t)} \longrightarrow-\infty
$$

it follows $\frac{f(t)}{m(t)} \leq f_{\mathrm{Max}} \forall t \geq 0$. Hence, we conclude that

$$
\frac{f(t)}{m(t)} \in\left[f_{\mathrm{Min}}, f_{\mathrm{Max}}\right] \forall t \geq 0 .
$$


The positivity of $b$ follows since $b(0)=0$ and $b(t)=0$ implies

$$
\frac{\mathrm{d}}{\mathrm{d} t} b(t) \stackrel{6}{=} k_{f} \frac{\left(f(t)-f_{\mathrm{Min}} m\right)^{2}}{f_{\mathrm{Max}} m-f(t)} \stackrel{14}{\geq} 0
$$

which finishes the proof of 1 . (It also follows that the time-dependent solution $(\ell(t), \tilde{\ell}(t), h(t), f(t), b(t), m(t)), t \geq 0$ of the initial value problem is unique and smooth because the smoothness of the right hand side of the ordinary differential equation (6) is now straight-forward to show.)

Using 14

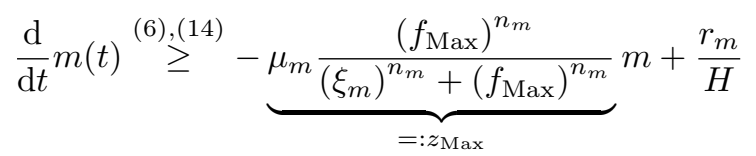

holds, and by solving this ordinary differential inequality with associated initial condition $m(0)=m_{0}$ it follows

$$
\begin{aligned}
m(t) & \stackrel{16}{\geq} m_{0} \exp \left(-z_{\mathrm{Max}} t\right)+\frac{r_{m}}{H z_{\mathrm{Max}}}\left(1-\exp \left(-z_{\mathrm{Max}} t\right)\right) \\
& \geq \min \left(m_{0}, \frac{r_{m}}{H z_{\mathrm{Max}}}\right) \stackrel{7}{ }, f_{\mathrm{Max}}>f_{0} \frac{r_{m}}{H z_{\mathrm{Max}}} \\
& =\frac{r_{m}}{H} \frac{\left(\xi_{m}\right)^{n_{m}}+\left(f_{\mathrm{Max}}\right)^{n_{m}}}{\mu_{m}\left(f_{\mathrm{Max}}\right)^{n_{m}}} \forall t \geq 0 .
\end{aligned}
$$

In an analogue way, the upper bound for $m(t)$ can be found, leading to

$$
m(t) \in[\underbrace{\frac{r_{m}}{H} \frac{\left(\xi_{m}\right)^{n_{m}}+\left(f_{\mathrm{Max}}\right)^{n_{m}}}{\mu_{m}\left(f_{\mathrm{Max}}\right)^{n_{m}}}}_{=: m_{\mathrm{Min}}}, \underbrace{\frac{r_{m}}{H} \frac{\left(\xi_{m}\right)^{n_{m}}+\left(f_{\mathrm{Min}}\right)^{n_{m}}}{\mu_{m}\left(f_{\mathrm{Min}}\right)^{n_{m}}}}_{=: m_{\mathrm{Max}}}] \forall t \geq 0
$$

which finishes the proof of 2 .

The boundedness of $\ell(t)$ is given by

$$
\frac{\mathrm{d}}{\mathrm{d} t} \ell(t) \stackrel{6}{=}-\left(q_{\ell, m} m_{\mathrm{Min}}+\frac{q_{\ell, e}}{H}\right) \ell(t)+\frac{r_{\ell}}{H}
$$

since the solution of the ordinary differential inequality (with associated initial condition $\ell(0)=0$ ) is bounded by

$$
\begin{aligned}
\ell(t) & \stackrel{19}{\leq} \frac{r_{\ell}}{H q_{\ell, m} m_{\mathrm{Min}}+q_{\ell, e}}\left(1-\exp \left(-\left(q_{\ell, m} m_{\mathrm{Min}}+\frac{q_{\ell, e}}{H}\right) t\right)\right) \\
& \leq \underbrace{\frac{r_{\ell}}{H q_{\ell, m} m_{\mathrm{Min}}+q_{\ell, e}}}_{\ell_{\operatorname{Max}}} .
\end{aligned}
$$


In an analogue way, the boundedness of $h(t)$ is proved. We show boundedness of $\tilde{\ell}(t)$ under the condition $\frac{r_{\ell}}{H}<\mu_{\tilde{\ell}} m_{\text {Min }}$ by a proof by contradiction. Hence, let $\tilde{\ell}(t)$ be unbounded, i.e. $\tilde{\ell}(t) \rightarrow \infty$ as $t \rightarrow \infty$ and $\frac{r_{\ell}}{H}<\mu_{\tilde{\ell}} m_{\text {Min }}$. It follows

$$
\begin{aligned}
\frac{\mathrm{d}}{\mathrm{d} t} \tilde{\ell}(t) \stackrel{[6,}{\stackrel{620}{\leq}} \underbrace{\left(-\mu_{\tilde{\ell}}+q_{\ell, m} \ell_{\mathrm{Max}}\right)}_{<0, \text { since } \frac{r_{\ell}}{H}<\mu_{\tilde{\ell}} m_{\mathrm{Min}}} m(t)+\frac{q_{\ell, e}}{H} \ell_{\mathrm{Max}} \\
\quad \\
\stackrel{18}{\leq}-\mu_{\tilde{\ell}} m_{\mathrm{Min}}+q_{\ell, m} \ell_{\mathrm{Max}} m_{\mathrm{Min}}+\frac{q_{\ell, e}}{H} \ell_{\mathrm{Max}}=-\mu_{\tilde{\ell}} m_{\mathrm{Min}}+\frac{r_{\ell}}{H}<0
\end{aligned}
$$

which is in contradiction to the assumed unboundedness of $\tilde{\ell}(t)$. Hence, this finishes the proof of Proposition 1.

\section{References}

1. Alon, R., Kassner, P.D., Carr, M.W., Finger, E.B., Hemler, M.E., Springer, T.A.: The integrin vla-4 supports tethering and rolling in flow on vcam-1. The Journal of Cell Biology 128(6), 1243-1253 (1995)

2. Assmann, G., Nofer, J.R.: Atheroprotective effects of high-density lipoproteins. Annual Review of Medicine 54(1), 321-341 (2003)

3. Brown, M.S., Goldstein, J.L.: Lipoprotein metabolism in the macrophage: implications for cholesterol deposition in atherosclerosis. Annual Review of Biochemistry 52(1) 223-261 (1983)

4. Brown, M.S., Goldstein, J.L., Krieger, M., Ho, Y., Anderson, R.: Reversible accumulation of cholesteryl esters in macrophages incubated with acetylated lipoproteins. The Journal of Cell Biology 82(3), 597-613 (1979)

5. Brown, M.S., Ho, Y., Goldstein, J.: The cholesteryl ester cycle in macrophage foam cells continual hydrolysis and re-esterification of cytoplasmic cholesteryl esters. Journal of Biological Chemistry 255(19), 9344-9352 (1980)

6. Brownell, N., Rohatgi, A.: Modulating cholesterol efflux capacity to improve cardiovascular disease. Current Opinion in Lipidology 27(4), 398-407 (2016)

7. Bulelzai, M.A., Dubbeldam, J.L.: Long time evolution of atherosclerotic plaques. Journal of Theoretical Biology 297, 1-10 (2012)

8. Calvez, V., Houot, J.G., Meunier, N., Raoult, A., Rusnakova, G.: Mathematical and numerical modeling of early atherosclerotic lesions. ESAIM: Proceedings and Surveys 30, 14 (2010). DOI $10.1051 / \mathrm{proc} / 2010002$

9. Chalmers, A.D., Cohen, A., Bursill, C.A., Myerscough, M.R.: Bifurcation and dynamics in a mathematical model of early atherosclerosis. Journal of Mathematical Biology 71(6-7), 1451-1480 (2015)

10. Chen, N., Frishman, W.H.: High-density lipoprotein infusion therapy and atherosclerosis: Current research and future directions. Cardiology in Review 24(6), 298-302 (2016)

11. Cobbold, C., Sherratt, J., Maxwell, S.: Lipoprotein oxidation and its significance for atherosclerosis: a mathematical approach. Bulletin of Mathematical Biology 64(1), 65-95 (2002)

12. Crosetto, P., Reymond, P., Deparis, S., Kontaxakis, D., Stergiopulos, N., Quarteroni, A.: Fluid-structure interaction simulation of aortic blood flow. Computers \& Fluids 43(1), 46-57 (2011)

13. Crouse, J., Parks, J., Schey, H., Kahl, F.: Studies of low density lipoprotein molecular weight in human beings with coronary artery disease. Journal of Lipid Research 26(5), 566-574 (1985)

14. Cybulsky, M.I., Gimbrone Jr, M.A.: Endothelial expression of a mononuclear leukocyte adhesion molecule during atherogenesis. Science 251(4995), 788 (1991) 
15. De Wilde, D., Trachet, B., De Meyer, G., Segers, P.: The influence of anesthesia and fluid-structure interaction on simulated shear stress patterns in the carotid bifurcation of mice. Journal of Biomechanics 49(13), 2741-2747 (2016)

16. DeVries-Seimon, T., Li, Y., Yao, P.M., Stone, E., Wang, Y., Davis, R.J., Flavell, R., Tabas, I.: Cholesterol-induced macrophage apoptosis requires er stress pathways and engagement of the type a scavenger receptor. Journal of Cell Biology 171(1), 61-73 (2005)

17. El Khatib, N., Génieys, S., Kazmierczak, B., Volpert, V.: Reaction-diffusion model of atherosclerosis development. Journal of Mathematical Biology 65(2), 349-374 (2012)

18. Faxon, D.P., Fuster, V., Libby, P., Beckman, J.A., Hiatt, W.R., Thompson, R.W., Topper, J.N., Annex, B.H., Rundback, J.H., Fabunmi, R.P., et al.: Atherosclerotic vascular disease conference writing group iii: Pathophysiology. Circulation 109(21), 2617-2625 (2004)

19. Feintuch, A., Ruengsakulrach, P., Lin, A., Zhang, J., Zhou, Y.Q., Bishop, J., Davidson, L., Courtman, D., Foster, F.S., Steinman, D.A., et al.: Hemodynamics in the mouse aortic arch as assessed by mri, ultrasound, and numerical modeling. American Journal of Physiology-Heart and Circulatory Physiology 292(2), H884-H892 (2007)

20. Feng, B., Yao, P.M., Li, Y., Devlin, C.M., Zhang, D., Harding, H.P., Sweeney, M., Rong, J.X., Kuriakose, G., Fisher, E.A., et al.: The endoplasmic reticulum is the site of cholesterol-induced cytotoxicity in macrophages. Nature Cell Biology 5(9), 781-792 (2003)

21. Friedman, A., Hao, W.: A mathematical model of atherosclerosis with reverse cholesterol transport and associated risk factors. Bulletin of Mathematical Biology 77(5), 758-781 (2015)

22. Frostegard, J., Haegerstrand, A., Gidlund, M., Nilsson, J.: Biologically modified ldl increases the adhesive properties of endothelial cells. Atherosclerosis 90(2-3), 119-126 (1991)

23. Glagov, S., Weisenberg, E., Zarins, C.K., Stankunavicius, R., Kolettis, G.J.: Compensatory enlargement of human atherosclerotic coronary arteries. New England Journal of Medicine 316(22), 1371-1375 (1987)

24. Gordon, S., Taylor, P.R.: Monocyte and macrophage heterogeneity. Nature Reviews Immunology 5(12), 953-964 (2005)

25. Hansson, G.K., Libby, P.: The immune response in atherosclerosis: a double-edged sword. Nature Reviews Immunology 6(7), 508-519 (2006)

26. of Health, N.I., National Heart, L., Institute, B., et al.: Atp iii guidelines at-a-glance quick desk reference. NIH publication (01-3305) (2001)

27. Henriksen, T., Mahoney, E.M., Steinberg, D.: Enhanced macrophage degradation of biologically modified low density lipoprotein. Arteriosclerosis, Thrombosis, and Vascular Biology 3(2), 149-159 (1983)

28. Ibragimov, A., McNeal, C., Ritter, L., Walton, J.: A mathematical model of atherogenesis as an inflammatory response. Mathematical Medicine and Biology 22(4), 305-333 (2005)

29. Jeng, J.R., Chang, C.H., Shih-Ming, S., Hui-Chong, C.: Oxidized low-density lipoprotein enhances monocyte-endothelial cell binding against shear-stress-induced detachment. Biochimica et Biophysica Acta (BBA)-Molecular Cell Research 1178(2), 221-227 (1993)

30. Kontush, A., Chapman, M.J.: Functionally defective high-density lipoprotein: a new therapeutic target at the crossroads of dyslipidemia, inflammation, and atherosclerosis. Pharmacological Reviews 58(3), 342-374 (2006)

31. Kunjathoor, V.V., Febbraio, M., Podrez, E.A., Moore, K.J., Andersson, L., Koehn, S., Rhee, J.S., Silverstein, R., Hoff, H.F., Freeman, M.W.: Scavenger receptors class ai/ii and $\operatorname{cd} 36$ are the principal receptors responsible for the uptake of modified low density lipoprotein leading to lipid loading in macrophages. Journal of Biological Chemistry 277(51), 49,982-49,988 (2002)

32. Leake, D.S., Rankin, S.M.: The oxidative modification of low-density lipoproteins by macrophages. Biochemical Journal 270(3), 741-748 (1990)

33. Leuschner, F., Rauch, P.J., Ueno, T., Gorbatov, R., Marinelli, B., Lee, W.W., Dutta, P., Wei, Y., Robbins, C., Iwamoto, Y., et al.: Rapid monocyte kinetics in acute myocardial infarction are sustained by extramedullary monocytopoiesis. Journal of Experimental Medicine 209(1), 123-137 (2012) 
34. Ley, K., Laudanna, C., Cybulsky, M.I., Nourshargh, S.: Getting to the site of inflammation: the leukocyte adhesion cascade updated. Nature Reviews Immunology $7(9)$, 678-689 (2007)

35. Libby, P.: Inflammation in atherosclerosis. Nature 420, 868-874 (2002)

36. Libby, P., Tabas, I., Fredman, G., Fisher, E.A.: Inflammation and its resolution as determinants of acute coronary syndromes. Circulation Research 114(12), 1867-1879 (2014)

37. Lin, J., Li, H., Yang, M., Ren, J., Huang, Z., Han, F., Huang, J., Ma, J., Zhang, D., Zhang, Z., et al.: A role of rip3-mediated macrophage necrosis in atherosclerosis development Cell Reports 3(1), 200-210 (2013)

38. Llodrá, J., Angeli, V., Liu, J., Trogan, E., Fisher, E.A., Randolph, G.J.: Emigration of monocyte-derived cells from atherosclerotic lesions characterizes regressive, but not progressive, plaques. Proceedings of the National Academy of Sciences of the United States of America 101(32), 11,779-11,784 (2004)

39. Lodish, H., Baltimore, D., Berk, A., Zipursky, S.L., Matsudaira, P., Darnell, J.: Molecular cell biology, vol. 3. Scientific American Books New York (1995)

40. Lougheed, M., Lum, C.M., Ling, W., Suzuki, H., Kodama, T., Steinbrecher, U.: High affinity saturable uptake of oxidized low density lipoprotein by macrophages from mice lacking the scavenger receptor class a type i/ii. Journal of Biological Chemistry 272(20), 12,938-12,944 (1997)

41. Mackness, M., Abbott, C., Arrol, S., Durrington, P.: The role of high-density lipoprotein and lipid-soluble antioxidant vitamins in inhibiting low-density lipoprotein oxidation. Biochemical Journal 294(3), 829-834 (1993)

42. Melmed, R., Karanian, P., Berlin, R.: Control of cell volume in the j774 macrophage by microtubule disassembly and cyclic amp. The Journal of Cell Biology 90(3), 761-768 (1981)

43. Milo, R.: What is the total number of protein molecules per cell volume? a call to rethink some published values. Bioessays 35(12), 1050-1055 (2013)

44. Moireau, P., Xiao, N., Astorino, M., Figueroa, C.A., Chapelle, D., Taylor, C.A., Gerbeau, J.F.: External tissue support and fluid-structure simulation in blood flows. Biomechanics and Modeling in Mechanobiology 11(1-2), 1-18 (2012)

45. Moore, K.J., Sheedy, F.J., Fisher, E.A.: Macrophages in atherosclerosis: a dynamic balance. Nature Reviews Immunology 13(10), 709-721 (2013)

46. Moore, K.J., Tabas, I.: Macrophages in the pathogenesis of atherosclerosis. Cell 145(3), 341-355 (2011)

47. Morel, D., DiCorleto, P.E., Chisolm, G.: Endothelial and smooth muscle cells alter low density lipoprotein in vitro by free radical oxidation. Arteriosclerosis, Thrombosis, and Vascular Biology 4(4), 357-364 (1984)

48. Mosser, D.M., Edwards, J.P.: Exploring the full spectrum of macrophage activation Nature Reviews Immunology 8(12), 958-969 (2008)

49. Nagano, Y., Arai, H., Kita, T.: High density lipoprotein loses its effect to stimulate efflux of cholesterol from foam cells after oxidative modification. Proceedings of the National Academy of Sciences 88(15), 6457-6461 (1991)

50. Ougrinovskaia, A., Thompson, R.S., Myerscough, M.R.: An ode model of early stages of atherosclerosis: mechanisms of the inflammatory response. Bulletin of Mathematical Biology 72(6), 1534-61 (2010). DOI 10.1007/s11538-010-9509-4

51. Parton, A., McGilligan, V., O'Kane, M., Baldrick, F.R., Watterson, S.: Computational modelling of atherosclerosis. Briefings in Bioinformatics p. bbv081 (2015)

52. Peiffer, V., Sherwin, S.J., Weinberg, P.D.: Does low and oscillatory wall shear stress correlate spatially with early atherosclerosis? a systematic review. Cardiovascular Research p. cvt044 (2013)

53. Phillips, M.C., Gillotte, K.L., Haynes, M.P., Johnson, W.J., Lund-Katz, S., Rothblat, G.H.: Mechanisms of high density lipoprotein-mediated efflux of cholesterol from cell plasma membranes. Atherosclerosis 137, S13-S17 (1998)

54. Potteaux, S., Gautier, E.L., Hutchison, S.B., van Rooijen, N., Rader, D.J., Thomas, M.J., Sorci-Thomas, M.G., Randolph, G.J.: Suppressed monocyte recruitment drives macrophage removal from atherosclerotic plaques of apoe- $/-$ mice during disease regression. The Journal of Clinical Investigation 121(5), 2025-2036 (2011) 
55. Prosi, M., Zunino, P., Perktold, K., Quarteroni, A.: Mathematical and numerical models for transfer of low-density lipoproteins through the arterial walls: a new methodology for the model set up with applications to the study of disturbed lumenal flow. Journal of Biomechanics 38(4), 903-917 (2005)

56. Rajman, I., Eacho, P.I., Chowienczyk, P., Ritter, J.: Ldl particle size: an important drug target? British Journal of Clinical Pharmacology 48(2), 125-133 (1999)

57. Robbins, C.S., Hilgendorf, I., Weber, G.F., Theurl, I., Iwamoto, Y., Figueiredo, J.L., Gorbatov, R., Sukhova, G.K., Gerhardt, L.M., Smyth, D., et al.: Local proliferation dominates lesional macrophage accumulation in atherosclerosis. Nature Medicine 19(9), 1166-1172 (2013)

58. Ross, R.: Atherosclerosis - an inflammatory disease. New England Journal of Medicine 340(2), 115-126 (1999)

59. Schrijvers, D.M., De Meyer, G.R., Kockx, M.M., Herman, A.G., Martinet, W.: Phagocytosis of apoptotic cells by macrophages is impaired in atherosclerosis. Arteriosclerosis, Thrombosis, and Vascular Biology 25(6), 1256-1261 (2005)

60. Soran, H., Schofield, J.D., Liu, Y., Durrington, P.N.: How hdl protects ldl against atherogenic modification: paraoxonase 1 and other dramatis personae. Current Opinion in Lipidology 26(4), 247-256 (2015)

61. Stary, H.C., Chandler, A.B., Dinsmore, R.E., Fuster, V., Glagov, S., Insull, W., Rosenfeld, M.E., Schwartz, C.J., Wagner, W.D., Wissler, R.W.: A definition of advanced types of atherosclerotic lesions and a histological classification of atherosclerosis. Circulation 92(5), 1355-1374 (1995)

62. Stary, H.C., Chandler, A.B., Glagov, S., Guyton, J.R., Insull, W., Rosenfeld, M.E., Schaffer, S.A., Schwartz, C.J., Wagner, W.D., Wissler, R.W.: A definition of initial, fatty streak, and intermediate lesions of atherosclerosis. a report from the committee on vascular lesions of the council on arteriosclerosis, american heart association. Circulation 89(5), 2462-2478 (1994)

63. Steingberg, D.: Beyond cholesterol modification of low density lipoprotein that increase its atherogenecity. New England Journal of Medicine 320, 915-924 (1989)

64. Stender, S., Zilversmit, D.: Transfer of plasma lipoprotein components and of plasma proteins into aortas of cholesterol-fed rabbits. molecular size as a determinant of plasma lipoprotein influx. Arteriosclerosis, Thrombosis, and Vascular Biology 1(1), 38-49 (1981)

65. Stocker, R., Keaney, J.F.: Role of oxidative modifications in atherosclerosis. Physiological Reviews 84(4), 1381-1478 (2004)

66. Tabas, I.: Consequences and therapeutic implications of macrophage apoptosis in atherosclerosis. Arteriosclerosis, Thrombosis, and Vascular Biology 25(11), 2255-2264 (2005)

67. Tabas, I.: 2016 russell ross memorial lecture in vascular biology. Arteriosclerosis, Thrombosis, and Vascular Biology 37(2), 183-189 (2017)

68. Tabas, I., Marathe, S., Keesler, G.A., Beatini, N., Shiratori, Y.: Evidence that the initial up-regulation of phosphatidylcholine biosynthesis in free cholesterol-loaded macrophages is an adaptive response that prevents cholesterol-induced cellular necrosis proposed role of an eventual failure of this response in foam cell necrosis in advanced atherosclerosis. Journal of Biological Chemistry 271(37), 22,773-22,781 (1996)

69. Teerlink, T., Scheffer, P.G., Bakker, S.J., Heine, R.J.: Combined data from ldl composition and size measurement are compatible with a discoid particle shape. Journal of Lipid Research 45(5), 954-966 (2004)

70. Tompkins, R.G.: Quantitative analysis of blood vessel permeability of squirrel monkeys. American Journal of Physiology - Heart and Circulatory Physiology 260(4), H1194H1204 (1991)

71. Véniant, M.M., Withycombe, S., Young, S.G.: Lipoprotein size and atherosclerosis susceptibility in apoe-/- and ldlr-/- mice. Arteriosclerosis, Thrombosis, and Vascular Biology 21(10), 1567-1570 (2001)

72. Virmani, R., Burke, A.P., Kolodgie, F.D., Farb, A.: Vulnerable plaque: the pathology of unstable coronary lesions. Journal of Interventional Cardiology 15(6), 439-446 (2002)

73. Wang, W., Lee, Y., Lee, C.H.: Review: The physiological and computational approaches for atherosclerosis treatment. International Journal of Cardiology 167(5), 1664-1676 (2013) 
74. Williams, K.J., Tabas, I.: The response-to-retention hypothesis of early atherogenesis. Arteriosclerosis, Thrombosis, and Vascular Biology 15(5), 551-561 (1995)

75. Witztum, J.L., Steinberg, D.: Role of oxidized low density lipoprotein in atherogenesis Journal of Clinical Investigation 88(6), 1785 (1991)

76. Yang, N., Vafai, K.: Modeling of low-density lipoprotein (ldl) transport in the arteryeffects of hypertension. International Journal of Heat and Mass Transfer 49(5), 850-867 (2006)

77. Yao, P.M., Tabas, I.: Free cholesterol loading of macrophages induces apoptosis involving the fas pathway. Journal of Biological Chemistry 275(31), 23,807-23,813 (2000)

78. Zhu, X., Lee, J.Y., Timmins, J.M., Brown, J.M., Boudyguina, E., Mulya, A., Gebre, A.K., Willingham, M.C., Hiltbold, E.M., Mishra, N., Maeda, N., Parks, J.S.: Increased cellular free cholesterol in macrophage-specific abca1 knock-out mice enhances pro-inflammatory response of macrophages. Journal of Biological Chemistry 283(34), 22,930-22,941 (2008)

79. Zi, Z.: Sensitivity analysis approaches applied to systems biology models. IET Systems Biology 5(6), 336-346 (2011)

80. Zohdi, T., Holzapfel, G., Berger, S.: A phenomenological model for atherosclerotic plaque growth and rupture. Journal of Theoretical Biology 227(3), 437-443 (2004) 